\title{
Restricted maximum-likelihood method for learning latent variance components in gene expression data with known and unknown confounders
}

\author{
Muhammad Ammar Malik and Tom Michoel ${ }^{*}$
}

May 6, 2020

Computational Biology Unit, Department of Informatics, University of Bergen, PO Box 7803, 5020 Bergen, Norway

* Corresponding author, email: tom.michoel@uib.no

\begin{abstract}
Linear mixed modelling is a popular approach for detecting and correcting spurious sample correlations due to hidden confounders in genome-wide gene expression data. In applications where some confounding factors are known, estimating simultaneously the contribution of known and latent variance components in linear mixed models is a challenge that has so far relied on numerical gradient-based optimizers to maximize the likelihood function. This is unsatisfactory because the resulting solution is poorly characterized and the efficiency of the method may be suboptimal. Here we prove analytically that maximumlikelihood latent variables can always be chosen orthogonal to the known confounding factors, in other words, that maximum-likelihood latent variables explain sample covariances not already explained by known factors. Based on this result we propose a restricted maximum-likelihood method which estimates the latent variables by maximizing the likelihood on the restricted subspace orthogonal to the known confounding factors, and show that this reduces to probabilistic PCA on that subspace. The method then estimates the variance-covariance parameters by maximizing the remaining terms in the likelihood function given the latent variables, using a newly derived analytic solution for this problem. Compared to gradient-based optimizers, our method attains equal or higher likelihood values, can be computed using standard matrix operations, results in latent factors that don't overlap with any known factors, and has a runtime reduced by several orders of magnitude. We anticipate that the restricted maximum-likelihood method will facilitate the application of linear mixed modelling strategies for learning latent variance components to much larger gene expression datasets than currently possible.
\end{abstract}

\section{Introduction}

Following the success of genome-wide association studies (GWAS) in mapping the genetic architecture of complex traits and diseases in human and model organisms [1-3], there is 
nowadays a great interest in complementing these studies with molecular data to understand how genetic variation affects epigenetic and gene expression states [4-6]. In GWAS, it is well-known that population structure or cryptic relatedness among individuals may lead to confouding that can alter significantly the outcome of the study [7]. When dealing with molecular data, this is further exacerbated by the often unknown technical or environmental influences on the data generating process. This problem is not confined to population-based studies - in single-cell analyses of gene expression, hidden subpopulations of cells and an even greater technical variability cause significant expression heterogeneity that needs to be accounted for [8].

In GWAS, linear mixed models have been hugely successful in dealing with confounding due to population structure [7,9-12]. In these models, it is assumed that an individual's trait value is a linear function of fixed and random effects, where the random effects are normally distributed with a covariance matrix determined by the genetic similarities between individuals, hence accounting for confounding in the trait data. Linear mixed models have also become popular in the correction for hidden confounders in gene expression data [13-15], generally outperforming approaches based on principal component analysis (PCA), the singular value decomposition or other hidden factor models [16-18]. In this context, estimating the latent factors and the sample-to-sample correlations they induce on the observed highdimensional data is the critical problem to solve.

If it is assumed that the observed correlations between samples are entirely due to latent factors, it can be shown that the resulting mixed model is equivalent to probabilistic PCA, which can be solved analytically in terms of the dominant eigenvectors of the sample covariance matrix $[19,20]$. However, in most applications, some confounding factors are known in advance (e.g. batch effects, genetic factors in population-based studies, or cell-cycle stage in single-cell studies), and the challenge is to estimate simultaneously the contribution of the known as well as the latent factors. This has so far relied on the use of numerical gradientbased optimizers to maximize the likelihood function $[8,15]$. This is unsatisfactory because the resulting solution is poorly characterized, the relation between the known and latent factors is obscured, and both the solution and the efficiency of the method may be suboptimal and dependent on the choice of initialization.

Intuitively, latent variables should explain sample covariances not already explained by known confounding factors. Here we demonstrate analytically that this intuition is correct: latent variables can always be chosen orthogonal to the known factors without reducing the likelihood or variance explained by the model. Based on this result we propose a method that is conceptually analogous to estimating fixed and random effects in linear mixed models using the restricted maximum-likelihood (REML) method, where the variance parameters of the random effects are estimated on the restricted subspace orthogonal to the maximumlikelihood estimates of the fixed effects [21]. Our method, called LVREML, similarly estimates the latent variables by maximizing the likelihood on the restricted subspace orthogonal to the known factors, and we show that this reduces to probabilistic PCA on that subspace. It then estimates the variance-covariance parameters by maximizing the remaining terms in the likelihood function given the latent variables, using a newly derived analytic solution for this problem. Similarly to the REML method for conventional linear mixed models, the LVREML solution is not guaranteed to maximize the total likelihood function. However we prove analytically that for any given number $p$ of latent variables, the LVREML solution attains minimal unexplained variance among all possible choices of $p$ latent variables, ar- 
guably a more intuitive and easier to understand criterion.

It is important to emphasize that our solution pertains to the estimation of the overall sampleto-sample covariance matrix to be used in the linear mixed model for expression heterogeneity, and not to the downstream estimation of variance parameters for individual genes given this covariance matrix. The latter remains a non-convex optimization problem for which numerical optimizers are required [11,12].

\section{Results}

\subsection{Restricted maximum-likelihood solution for a linear mixed model with known and latent variance components}

Our initial model to infer latent variance components in a gene expression data matrix is the same model that was popularized in the widely used PANAMA [15] and scLVM [8] softwares, where a linear relationship is assumed between expression levels and the known and latent factors, with random noise added (SI Section S2). In matrix notation, the model can be written as

$$
\mathbf{Y}=\mathbf{Z V}+\mathbf{X W}+\boldsymbol{\epsilon}
$$

where $\mathbf{Y} \in \mathbb{R}^{n \times m}$ is a matrix of gene expression data for $m$ genes in $n$ samples, and $\mathbf{Z} \in \mathbb{R}^{n \times d}$ and $\mathbf{X} \in \mathbb{R}^{n \times p}$ are matrices of values for $d$ known and $p$ latent confounders in the same $n$ samples. The columns $v_{i}$ and $w_{i}$ of the random matrices $\mathbf{V} \in \mathbb{R}^{d \times m}$ and $\mathbf{W} \in \mathbb{R}^{p \times m}$ are the effects of the known and latent confounders, respectively, on the expression level of gene $i$, and are assumed to be jointly normally distributed:

$$
p\left(\left[\begin{array}{c}
v_{i} \\
w_{i}
\end{array}\right]\right)=\mathcal{N}\left(0,\left[\begin{array}{cc}
\mathbf{B} & \mathbf{D} \\
\mathbf{D}^{T} & \mathbf{A}
\end{array}\right]\right)
$$

where $\mathbf{B} \in \mathbb{R}^{d \times d}, \mathbf{A} \in \mathbb{R}^{p \times p}$ and $\mathbf{D} \in \mathbb{R}^{d \times p}$ are the covariances of the known-known, latentlatent and known-latent confouder effects, respectively. Lastly, $\boldsymbol{\epsilon} \in \mathbb{R}^{n \times m}$ is a matrix of independent samples of a Gaussian distribution with mean zero and variance $\sigma^{2}$, independent of the confounding effects.

Previously, this model was considered with independent random effects (B and A diagonal and $\mathbf{D}=0)[8,15]$. As presented here, the model is more general and accounts for possible lack of independence between the effects of known covariates. Furthermore, allowing the effects of the known and latent factors to be dependent $(\mathbf{D} \neq 0)$ is precisely what will allow the latent variables to be orthogonal to the known confounders (SI Section S6). An equivalent model with $\mathbf{D}=0$ can be considered, but requires non-orthogonal latent variables to explain part of the sample covariance matrix, resulting in a mathematically less tractable framework. Finally, it remains the case that we can always choose $\mathbf{A}$ to be diagonal, because the latent factors have an inherent rotational symmetry that allows any non-diagonal model to be converted to an equivalent diagonal model (SI Section S5). By definition, the known covariates correspond to measured or "natural" variables, and hence they have no such rotational symmetry.

Using standard mixed-model calculations to integrate out the random effects (SI Section S2), the log-likelihood of the unknown model parameters given the observed data can be written 


$$
\mathcal{L}\left(\mathbf{X}, \mathbf{A}, \mathbf{B}, \sigma^{2} \mid \mathbf{Y}, \mathbf{Z}\right)=-\log \operatorname{det}(\mathbf{K})-\operatorname{tr}\left(\mathbf{K}^{-1} \mathbf{C}\right),
$$

where

$$
\mathbf{K}=\mathbf{Z} \mathbf{B} \mathbf{Z}^{T}+Z \mathbf{D} \mathbf{X}^{T}+\mathbf{X D}^{T} \mathbf{Z}^{T}+\mathbf{X} \mathbf{A} \mathbf{X}^{T}+\sigma^{2} \mathbb{1},
$$

and $\mathbf{C}=\left(\mathbf{Y} \mathbf{Y}^{T}\right) / m$ is the empirical sample covariance matrix. Maximizing the log-likelihood (2) over positive definite matrices $\mathbf{K}$ without any further constraints would result in the estimate $\hat{\mathbf{K}}=\mathbf{C}$ (note that $\mathbf{C}$ is invertible because we assume that the number of genes $m$ is greater than the number of samples $n$ ) [22].

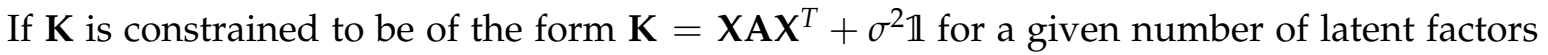
$p<n$, then the model is known as probabilistic PCA, and the likelihood is maximized by identifying the latent factors with the eigenvectors of $\mathbf{C}$ corresponding to the $p$ largest eigenvalues $[19,20]$. In matrix form, the probabilistic PCA solution can be written as

$$
\hat{\mathbf{K}}=\mathbf{P}_{1} \mathbf{C} \mathbf{P}_{1}+\hat{\sigma}^{2} \mathbf{P}_{2}
$$

where $\mathbf{P}_{1}$ and $\mathbf{P}_{2}$ are mutually orthogonal projection matrices on the space spanned by the first $p$ and last $n-p$ eigenvectors of $\mathbf{C}$, respectively, and the maximum-likelihood estimate $\hat{\sigma}^{2}$ is the average variance explained by the $n-p$ excluded dimensions (SI Section S5).

If $\mathbf{K}$ is constrained to be of the form $\mathbf{K}=\mathbf{Z B Z} \mathbf{Z}^{T}+\sigma^{2} \mathbb{1}$, the model is a standard linear mixed model with the same design matrix $\mathbf{Z}$ for the random effects $v_{i}$ for each gene $i$. In general, there exists no analytic solution for the maximum-likelihood estimates of the (co)variance parameter matrix $\mathbf{B}$ in a linear mixed model [21]. However in the present context it is assumed that the data for each gene is an independent sample of the same mixed model. Again using the fact that $\mathbf{C}=\left(\mathbf{Y} \mathbf{Y}^{T}\right) / m$ is invertible due to the number of genes being greater than the number of samples, the maximum-likelihood solution for $\mathbf{B}$, and hence $\mathbf{K}$, can be found analytically in terms of $\mathbf{C}$ and the singular value decomposition (SVD) of $\mathbf{Z}$. It turns out to be of the same form (4), except that $\mathbf{P}_{1}$ now projects onto the subspace spanned by the known covariates (the columns of Z) (SI Section S4).

In the most general case where $\mathbf{K}$ takes the form (3), we show first that every model of the form (1) can be rewritten as a model of the same form where the hidden factors are orthogonal to the known covariates, $\mathbf{X}^{T} \mathbf{Z}=0$. The reason is that any overlap between the hidden and known covariates can be absorbed in the random effects $v_{i}$ by a linear transformation, and therefore simply consists of a reparameterization of the covariance matrices $\mathbf{B}$ and $\mathbf{D}$ (SI Section S6.1). Once this orthogonality is taken into account, the log-likelihood (2) decomposes as a sum $\mathcal{L}=\mathcal{L}_{1}+\mathcal{L}_{2}$, where $\mathcal{L}_{2}$ is identical to the log-likelihood of probabilistic PCA on the reduced space that is the orthogonal complement to the subspace spanned by the known covariates (columns of $\mathbf{Z}$ ). Analogous to the restricted maximum-likelihood (REML) method for ordinary linear mixed models, where variance parameters of the random effects are estimated in the subspace orthogonal to the maximum-likelihood estimates of the fixed effects $[21,23]$, we estimate the latent variables $\boldsymbol{X}$ by maximizing only the likelihood term $\mathcal{L}_{2}$ corresponding to the subspace where these $\mathbf{X}$ live (Section S6.2). Once the restricted maximum-likelihood estimates $\hat{\mathbf{X}}$ are determined, they become "known" covariates, allowing the covariance parameter matrices to be determined by maximizing the remaining terms $\mathcal{L}_{1}$ in the likelihood function using the analytic solution for a model with known covariates $(\mathbf{Z}, \hat{\mathbf{X}})$ (Section S6.3). 
A

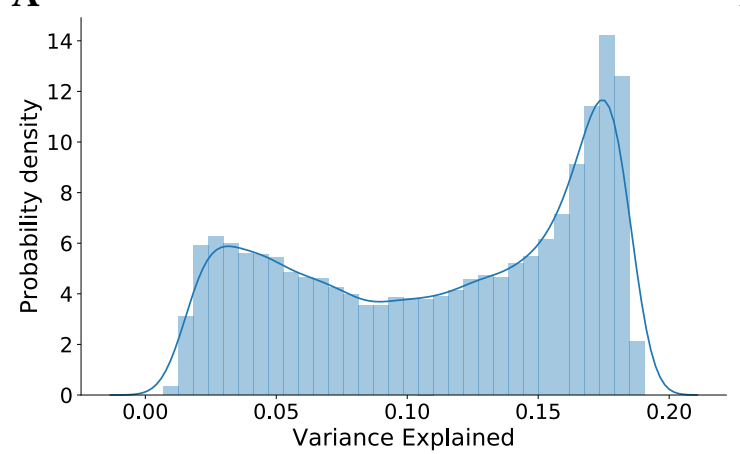

C

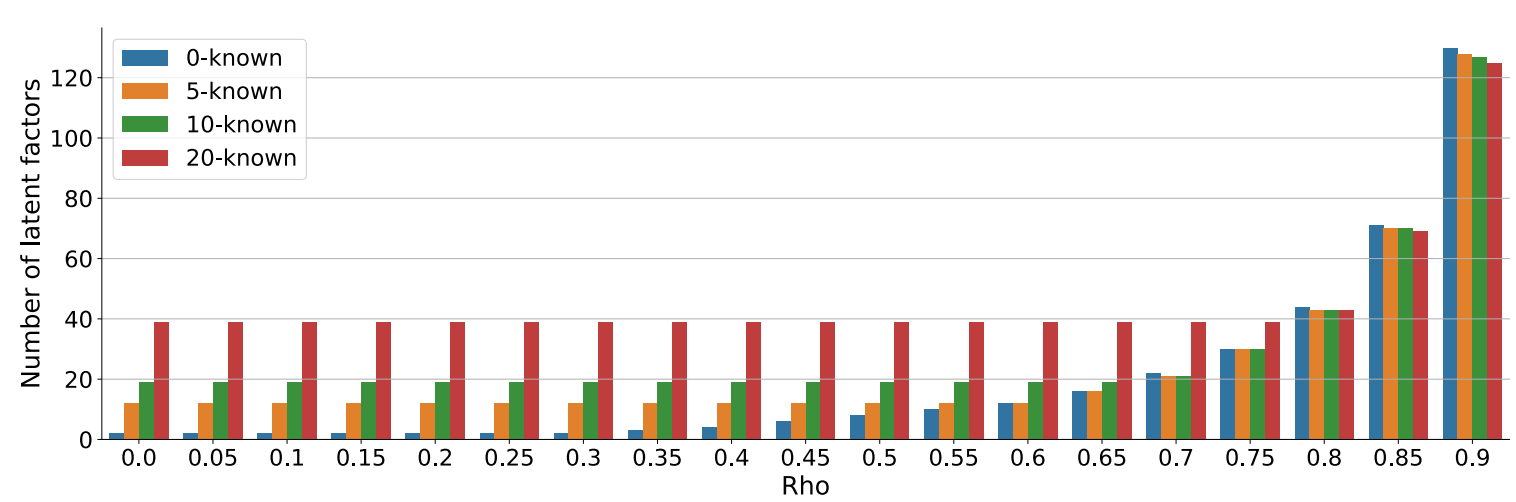

B

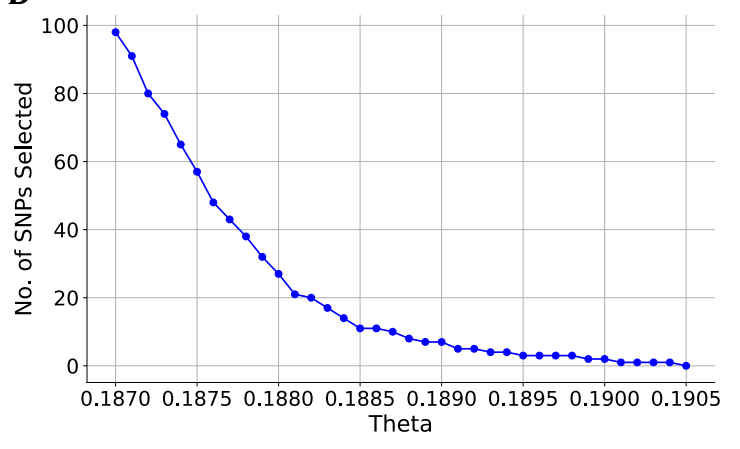

Figure 1: A. Distribution of the expression variance explained by individual SNPs in the Human Liver Cohort. B. Number of linearly independent SNPs selected as covariates as a function of the parameter $\theta$, the minimum variance explained by each covariate. C. Number of hidden covariates inferred by LVREML as a function of the parameter $\rho$, the total amount of variance explained by the known and latent variables, with $\theta$ set to have $0,5,10$, or 20 known covariates in the model.

By analogy with the REML method, we call our method the restricted maximum-likelihood method for solving the latent variable model (1), abbreviated "LVREML". While the LVREML solution is not guaranteed to be the absolute maximizer of the total likelihood function, it is guaranteed analytically that for any given number $p$ of latent variables, the LVREML solution attains minimal unexplained variance among all possible choices of $p$ latent variables (SI Section S6.4).

\subsection{LVREML, a flexible software package for learning latent variance components in gene expression data}

We implemented the restricted maximum-likelihood method for solving model (1) in a software package LVREML, available with Matlab and Python interfaces at https://github. $\mathrm{com} / \mathrm{michoel-lab/lvreml.} \mathrm{LVREML} \mathrm{takes} \mathrm{as} \mathrm{input} \mathrm{a} \mathrm{gene} \mathrm{expression} \mathrm{matrix} \mathbf{Y}$, a covariate matrix $\mathbf{Z}$, and a parameter $\rho$, with $0<\rho<1$. This parameter is the desired proportion of variation in $\mathbf{Y}$ that should be explained by the combined known and latent variance compo- 
nents. Given $\rho$, the number of latent factors $p$ is determined automatically (SI Section S7). LVREML centres the data $\mathbf{Y}$ such that each sample has mean value zero, to ensure that no fixed effects on the mean need to be included in the model (SI Section S3).

When the number of known covariates (or more precisely the rank of $\mathbf{Z}$ ) exceeds the number of samples, as happens in eQTL studies where a large number of SNPs can act as covariates [15], a subset of $n$ linearly independent covariates will always explain all of the variation in $\mathbf{Y}$. In [15], a heuristic approach was used to select covariates during the likelihood optimization, making it difficult to understand a priori which covariates will be included in the model and why. In contrast, LVREML includes a function to perform initial screening of the covariates, solving for each one the model (1) with a single known covariate to compute the variance $\hat{\beta}^{2}$ explained by that covariate alone (SI Section S4). This estimate is then used to include in the final model only those covariates for which $\hat{\beta}^{2} \geq \theta \operatorname{tr}(\mathbf{C})$, where $\theta>0$ is the second free parameter of the method, namely the minimum amount of variation a known covariate needs to explain on its own to be included in the model (SI Section S7).

To illustrate the effect of these parameters, we used data from the Human Liver Cohort (HLC) [24]. After removing SNPs and genes with missing data, expression data for 31,178 genes and genotype data for 49,879 SNPs in 178 publicly available samples were used for the analysis (Methods Section 4.2). Individual SNPs explained upto 19\% of the variation in $Y$, according to the single-covariate model (SI Section S4, eq. (S15)) (Fig. 1A). To illustrate how the number of inferred hidden covariates varies as a function of the input parameter $\rho$, we determined values of the parameter $\theta$ to include between 0 and 20 SNPs as covariates in the model (Fig. 1B). As expected, for a fixed number of known covariates, the number of hidden covariates increases with $\rho$, as more covariates are needed to explain more of the variation in $\mathbf{Y}$ (Fig. 1C).

Perhaps counterintuitively, for a fixed number of known covariates, there is a threshold for $\rho$ below which the inferred number of hidden covariates does not change; moreover both the threshold and the number of hidden covariates are higher when more known covariates are included. This is due to a mathematical property of the model: the maximizing solution exists only if the minimum amount of variation in $\mathbf{Y}$ explained by a principal axis in the space spanned by the known covariates is greater than the maximum-likelihood estimate of the residual variance $\hat{\sigma}^{2}$ (see Theorems 1 and 4 in SI Sections S4 and S6). If non-informative variables are included among the known covariates, then the minimum variation explained by them becomes small, and potentially smaller than the residual variance, whose initial 'target' value is $1-\rho$. Because LVREML considers the known covariates as fixed, it lowers the value of $\hat{\sigma}^{2}$ by including more hidden covariates in the model, until the existence condition is satisfied. Hence, in Fig. 1C, with 5 known covariates, LVREML cannot build a model that explains less than $65 \%$ of the variation in $Y$, without violating the existence condition, whereas with the 48 known covariates this increases to $85 \%$ of the variation. Hence it is important to run the initial covariate screening function to avoid including known covariates that explain only a very small proportion of variation in the expression data. 
A

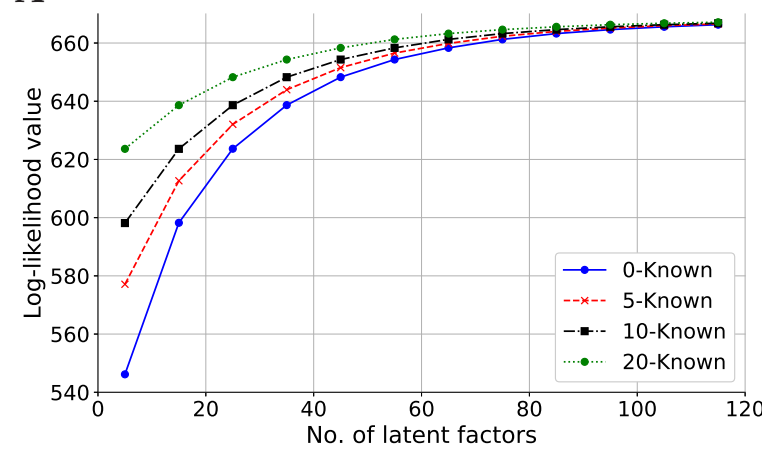

C

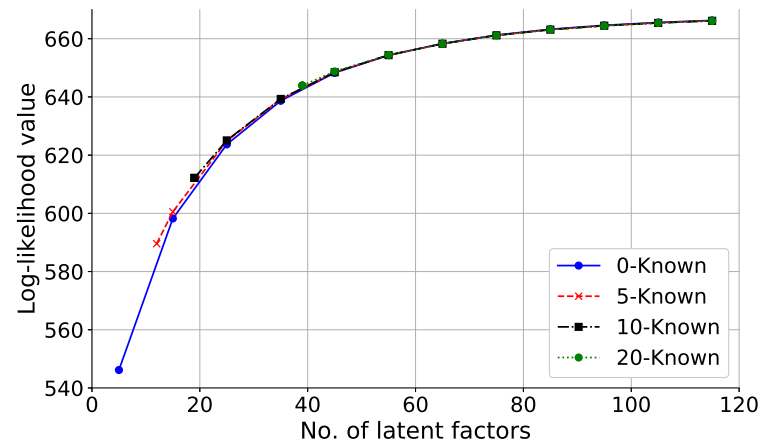

B

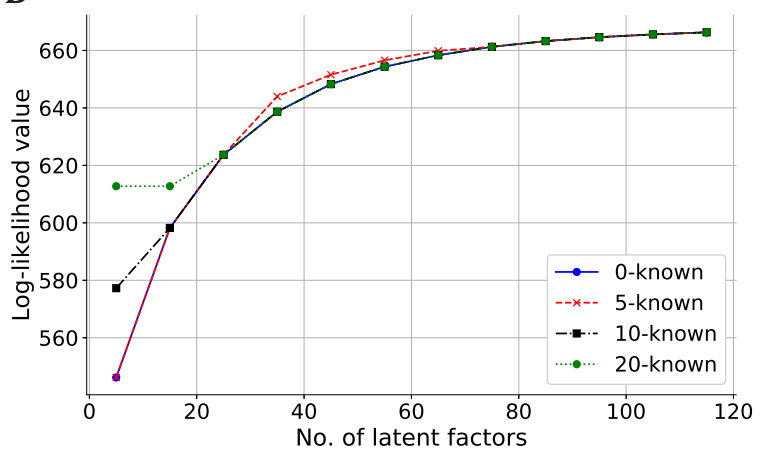

D

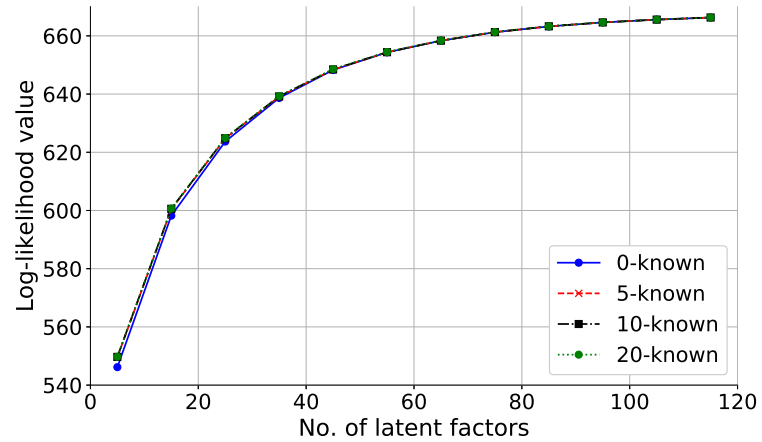

Figure 2: Log-likelihood values for LVREML (A,C) and PANAMA (B,D) using 0, 5, 10, and 20 principal components of the expression data $(\mathbf{A}, \mathbf{B})$, and sets of linearly independent SNPs $(\mathbf{C}, \mathbf{D})$ as known covariates, using data from the Human Liver Cohort.

\subsection{LVREML infers hidden factors that are non-overlapping with known covari- ates, and attains likelihood values higher than or equal to PANAMA}

To compare the analytic solution of LVREML against the original model with gradient-based optimization algorithm, we performed a controlled comparison using the PANAMA software [15]. We first calculated the principal components (PCs) of the HLC expression data, and then ran both LVREML and PANAMA using the first $0,5,10$, and 20 PCs as known covariates. Because of the mathematical properties of the model and the LVREML solution, if the first $d$ PCs are included as known covariates, LVREML will return the next $p$ PCs as hidden factors. Hence the log-likelihood of the LVREML solution with $d$ PCs as known covariates and $p$ hidden factors will coincide with the log-likelihood of the solution with zero known covariates and $d+p$ hidden factors (that is, probabilistic PCA with $d+p$ hidden factors). Fig. 2A shows that this is the case indeed: the log-likelihood curves for $0,5,10$, and 20 PCs as known covariates are shifted horizontally by a difference of exactly 5 (from 0 , to 5 , to 10 ) or 10 (from 10 to 20 ) hidden factors.

In contrast, PANAMA did not find the optimal shifted probabilistic PCA solution, and its likelihood values largely coincided with the solution with zero known covariates, irrespective of the number of known covariates provided (Fig. 2B). In other words, PANAMA did not use the knowledge of the known covariates to explore the orthogonal space of axes of variation not yet explained by the known covariates, instead arriving at a solution where $p$ hidden factors 
A

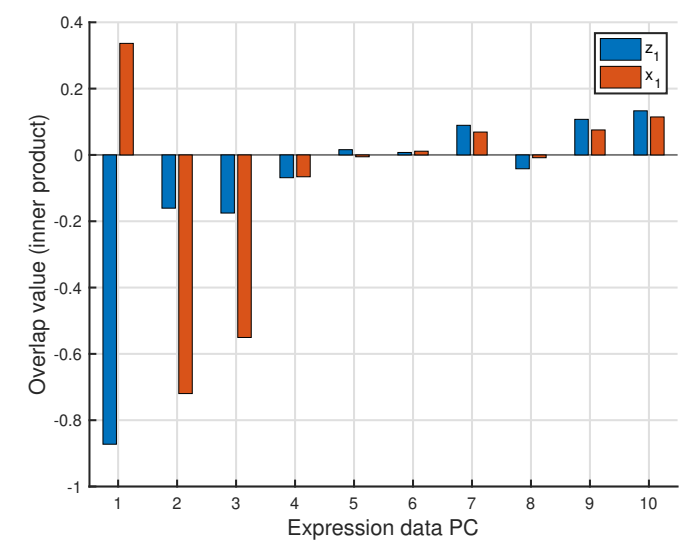

B

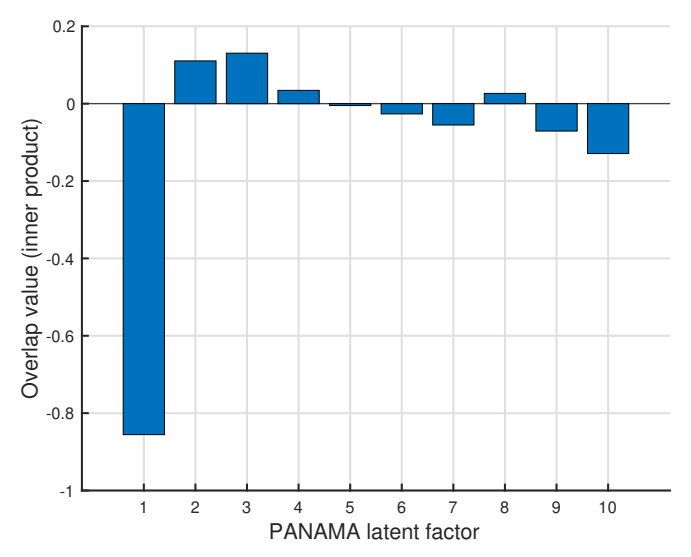

Figure 3: Overlap values (inner products) between the first 10 expression data PCs and the first known (SNP) and latent covariate inferred by LVREML (A), and between the first 10 PANAMA latent factors and the first known (SNP) covariate (B). Results are from the application of LVREML and PANAMA on the Human Liver Cohort with 5 known SNP covariates and 20 latent variables.

appear to explain no more of the variation than $p-d$ PCs orthogonal to the $d$ known PCs. To verify this, we compared the PANAMA hidden factors to PCs given as known covariates, and found that in all cases where the curves in Fig. 2B align, the first $d$ hidden factors coincided indeed with the $d$ PCs used as known covariates.

When SNPs were used as known confounders (using the procedure explained in Section 2.2), the log-likelihood values did not show the same clear dependence on the number of known covariates, and did not differ much between the two methods (Fig. 2C,D). The explanation is that the selected SNPs all overlapped most strongly with the first PC of the expression data (Fig. 3A), such that in terms of variance explained, and when comparing Fig. $2 \mathrm{C}$ to Fig. 2A, less than one effective degree of freedom is gained by including the SNPs as known covariates, and the 'shifted' log-likelihood curves become nearly indistinguishable from the probabilistic PCA curve. However, it is still the case that the latent variables inferred by LVREML represent orthogonal degrees of freedom to the known SNP covariates. In other words, the dominant PCs represent the main axes of variation in the expression data, and are partially explained by population structure (genotype data). The LVREML solution then infers orthogonal latent variables that explain the 'missing' portions of the PCs not explained by genotype data (Fig. 3A).

In contrast, the latent variables inferred by PANAMA again overlapped with the known SNP covariates supplied to the model (Fig. 3B). In the PANAMA model, covariances among the effects of the known confounders are assumed to be zero. When these effects in fact have nonzero covariance, the optimization algorithm will automatically select hidden confounders that overlap with the known confounders in order to account for these non-zero covariances (SI Section S6.1), thus resulting in the observed overlap. Hence the common interpretation of PANAMA factors as new determinants of gene expression distinct from known genetic factors is problematic. 
A

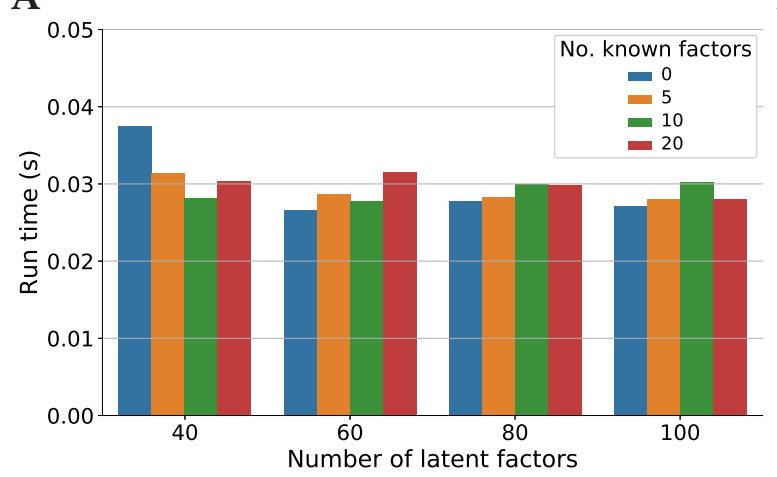

B

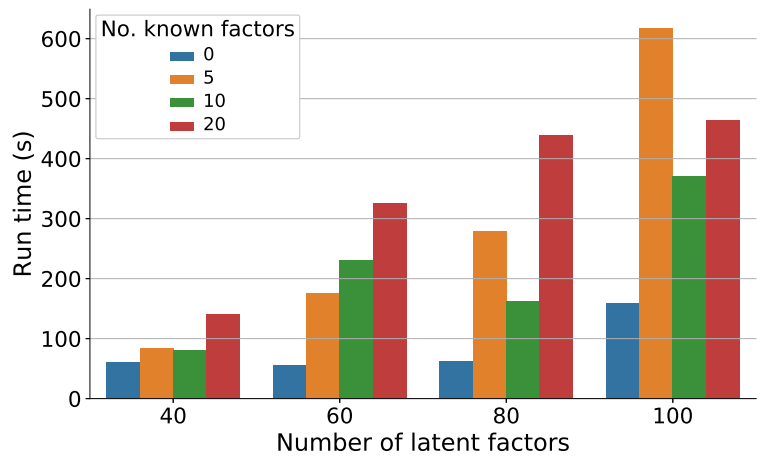

Figure 4: Runtime comparison on the Human Liver Cohort between LVREML (A) and PANAMA (B) for the same parameter settings (number of known/latent factors).

\subsection{LVREML is orders of magnitude faster than PANAMA}

An analytic solution does not only provide additional insight into the mathematical properties of a model, but can also provide significant gains in computational efficiency. The LVREML solution can be computed using standard matrix operations from linear algebra, for which highly optimized implementations exist in all commonly used programming languages. Comparison of the runtime of the Python implementations of LVREML and PANAMA on the HLC data showed around ten thousand-fold speed-up factors, from several minutes for a single PANAMA run to a few tens of milliseconds for LVREML (Fig. 4). Moreover the runtime of LVREML does not depend on the number of known or inferred latent factors, whereas increasing either parameter in PANAMA leads to an increase in runtime (Fig. 4).

\section{Discussion}

We presented a linear mixed model to estimate simultaneously the contribution of known and latent variance components in gene expression data, which is closely related to models that have been used previously in this context $[8,15,17,18,20]$. By including additional parameters in our model to account for non-zero covariances among the effects on gene expression of known covariates and latent factors, we were able to show that latent factors can always be taken orthogonal to, and therefore linearly independent of, the known covariates supplied to the model. This is important, because inferred latent factors are not only used to correct for correlation structure in the data, but also as new, data-derived 'phenotypes', that is, determinants of gene expression whose own genetic associations are biologically informative [18]. As shown in this paper, the existing models and their numerical optimization result in hidden factors that in fact overlap with the known covariates, and hence their value in uncovering 'new' determinants of gene expression must be questioned.

To solve our model, we did not rely on numerical, gradient-based optimizers, but rather on an analytic restricted maximum-likelihood solution. This solution is based on a decomposition of the log-likelihood function that allows to identify hidden factors as principal components of the expression data matrix reduced to the orthogonal complement of the 
subspace spanned by the known covariates. This solution is guaranteed to minimize the amount of unexplained variation in the expression data for a given number of latent factors, and is analogous to the widely used restricted maximum-likelihood solution for conventional linear mixed models, where variance parameters of random effects are estimated in the subspace orthogonal to the maximum-likelihood estimates of the fixed effects.

Having an analytic solution is not only important for understanding the mathematical properties of a statistical model, but can also lead to significant reduction of the computational cost for estimating parameter values. Here, even on a small dataset with 178 samples, we obtained a ten-thousand fold speed-up compared to an existing software that uses gradientbased optimization.

To conclude, we have derived an analytic restricted maximum-likelihood solution for a widely used class of linear mixed models for learning latent variance components in gene expression data with known and unknown confounders. Our solution can be computed in a highly efficient manner, identifies hidden factors that are orthogonal to the already known variance components, and results in the estimation of a sample covariance matrix that can be used for the downstream estimation of variance parameters for individual genes. We anticipate that the restricted maximum-likelihood method will facilitate the application of linear mixed modelling strategies for learning latent variance components to much larger gene expression datasets than currently possible.

\section{Methods}

\subsection{Mathematical methods}

All model equations, mathematical results and detailed proofs are described in a separate Supplementary Information document.

\subsection{Data}

We used publicly available genotype and gene expression data from the Human Liver Cohort [24], downloaded from https://www.synapse.org/\#! Synapse: syn4499, and consisting of gene expression data for 40,638 genes in 466 individuals and genotype data for 449,698 SNPs in 195 individuals; 178 individuals had both expression and genotype data and were used in our analyses. We removed all genes and SNPs with missing data, and further removed SNPs with minor allele frequence less than $5 \%$ and that were not in Hardy-Weinberg equilibrium $\left(P<10^{-6}\right)$. This resulted in gene expression data for 31,178 genes and genotype data for 49,879 SNPs in 178 samples. Finally, gene expression samples were centred to have mean zero, and genotype samples were normalized to have unit L2-norm.

\subsection{LVREML analyses}

The LVREML software, as well as a script that details the LVREML analyses of the HLC data is available at https://github.com/michoel-lab/lvreml. 


\subsection{PANAMA analyses}

We obtained the PANAMA software from the LIMIX package available at https://github. com/limix/limix-legacy.

The following settings were used to ensure that exactly the same normalized data was used by both methods: 1) For parameter $\mathbf{Y}$, the same gene expression matrix, with each sample normalized to have zero mean, was used that was used as input for LVREML, setting the standardize parameter to false. 2) The parameter Ks requires a list of covariance matrices for each known factor. Therefore for each column $z_{i}$ of the matrix $\mathbf{Z}$ used by LVREML we included the covariance matrix $\mathbf{K s}_{i}=z_{i} z_{i}^{T}$ in $\mathbf{K s}$. The 'use Kpop' parameter, which is used to supply a population structure covariance matrix to PANAMA in addition to the known covariates, was set to false.

To be able to calculate the log-likelihoods and extract other relevant information from the PANAMA results, we made the following modifications to the PANAMA code: 1) The covariance matrices returned by PANAMA are by default normalized by dividing the elements of the matrix by the mean of its diagonal elements. To make these covariance matrices comparable to LVREML this normalization was omitted by commenting out the lines in the original PANAMA code where this normalization was being performed. 2) PANAMA does not return the variance explained by the known confounders unless the 'use Kpop' parameter is set to true. Therefore the code was modified so that it would still return the variance explained by the known confounders. 3) The $\mathbf{K}$ matrix returned by PANAMA does not include the effect of the noise parameter $\sigma^{2}$. Therefore the code was modified to return the $\sigma^{2} \mathbb{1}$ matrix, which was then added to the returned $\mathbf{K}$ i.e. $\mathbf{K}_{\text {new }}=\mathbf{K}+\sigma^{2} \mathbb{1}$, to be able to use equation (2) to compute the log-likelihood. The modified code is available as a fork of the LIMIX package at https://github.com/michoel-lab/limix-legacy

\section{References}

[1] Mackay TF, Stone EA and Ayroles JF. The genetics of quantitative traits: challenges and prospects. Nature Reviews Genetics 10:565-577 (2009).

[2] Hindorff LA et al. Potential etiologic and functional implications of genome-wide association loci for human diseases and traits. Proceedings of the National Academy of Sciences 106:9362-9367 (2009).

[3] Manolio TA. Bringing genome-wide association findings into clinical use. Nature Reviews Genetics 14:549-558 (2013).

[4] Albert FW and Kruglyak L. The role of regulatory variation in complex traits and disease. Nature Reviews Genetics 16:197-212 (2015).

[5] Franzén $\mathrm{O}$ et al. Cardiometabolic risk loci share downstream cis and trans genes across tissues and diseases. Science (2016).

[6] GTEx Consortium. Genetic effects on gene expression across human tissues. Nature 550:204 (2017). 
[7] Astle W, Balding DJ et al. Population structure and cryptic relatedness in genetic association studies. Statistical Science 24:451-471 (2009).

[8] Buettner F et al. Computational analysis of cell-to-cell heterogeneity in single-cell RNAsequencing data reveals hidden subpopulations of cells. Nature Biotechnology 33:155-160 (2015).

[9] Yu J et al. A unified mixed-model method for association mapping that accounts for multiple levels of relatedness. Nature Genetics 38:203 (2006).

[10] Kang HM et al. Variance component model to account for sample structure in genomewide association studies. Nature Genetics 42:348-354 (2010).

[11] Lippert C et al. FaST linear mixed models for genome-wide association studies. Nature Methods 8:833-835 (2011).

[12] Zhou X and Stephens M. Genome-wide efficient mixed-model analysis for association studies. Nature Genetics 44:821-824 (2012).

[13] Kang HM, Ye C and Eskin E. Accurate discovery of expression quantitative trait loci under confounding from spurious and genuine regulatory hotspots. Genetics 180:19091925 (2008).

[14] Listgarten $\mathrm{J}$ et al. Correction for hidden confounders in the genetic analysis of gene expression. Proceedings of the National Academy of Sciences 107:16465-16470 (2010).

[15] Fusi N, Stegle O and Lawrence ND. Joint modelling of confounding factors and prominent genetic regulators provides increased accuracy in genetical genomics studies. PLoS Computational Biology 8:e1002330 (2012).

[16] Leek JT and Storey JD. Capturing heterogeneity in gene expression studies by surrogate variable analysis. PLoS Genetics 3:e161 (2007).

[17] Stegle O et al. A Bayesian framework to account for complex non-genetic factors in gene expression levels greatly increases power in eQTL studies. PLoS computational biology 6:e1000770 (2010).

[18] Stegle O et al. Using probabilistic estimation of expression residuals (peer) to obtain increased power and interpretability of gene expression analyses. Nature Protocols 7:500507 (2012).

[19] Tipping ME and Bishop CM. Probabilistic principal component analysis. Journal of the Royal Statistical Society: Series B (Statistical Methodology) 61:611-622 (1999).

[20] Lawrence N. Probabilistic non-linear principal component analysis with gaussian process latent variable models. Journal of Machine Learning Research 6:1783-1816 (2005).

[21] Gumedze F and Dunne T. Parameter estimation and inference in the linear mixed model. Linear Algebra and its Applications 435:1920-1944 (2011).

[22] Anderson TW and Olkin I. Maximum-likelihood estimation of the parameters of a multivariate normal distribution. Linear algebra and its applications 70:147-171 (1985). 
[23] Patterson HD and Thompson R. Recovery of inter-block information when block sizes are unequal. Biometrika 58:545-554 (1971).

[24] Schadt EE et al. Mapping the genetic architecture of gene expression in human liver. PLoS Biol 6:e107 (2008).

[25] Horn RA and Johnson CR. Matrix analysis (Cambridge University Press1985). 


\section{Supplementary Information}

\section{S1 Preliminary results}

In the sections below, we will repeatedly use the following results. The first result concerns linear transformations of normally distributed variables and can be found in most textbooks on statistics or probability theory:

Lemma 1. Let $x \in \mathbb{R}^{n}$ be a random, normally distributed vector,

$$
p(x)=\mathcal{N}(\mu, \Psi)
$$

with $\mu \in \mathbb{R}^{n}$, and $\Psi \in \mathbb{R}^{n \times n}$ a positive definite covariance matrix. For any linear transformation $y=\mathbf{M} x$ with $\mathbf{M} \in \mathbb{R}^{m \times n}$, we have

$$
p(y)=\mathcal{N}\left(\mathbf{M} \mu, \mathbf{M} \Psi \mathbf{M}^{T}\right) .
$$

The second result is one that is attributed to von Neumann [22]:

Lemma 2. Let $\mathbf{P}, \mathbf{Q} \in \mathbb{R}^{n \times n}$ be two positive definite matrices. Then

$$
\operatorname{tr}\left(\mathbf{P}^{-1} \mathbf{Q}\right) \geq \sum_{i=1}^{n} \pi_{i}^{-1} \chi_{i}
$$

where $\pi_{1} \geq \cdots \geq \pi_{n}$ and $\chi_{1} \geq \cdots \geq \chi_{n}$ are the ordered eigenvalues of $\mathbf{P}$ and $\mathbf{Q}$, respectively, and equality in eq. (S1) is achieved if and only if the eigenvector of $\mathbf{P}$ corresponding to $\pi_{i}$ is equal to the eigenvector of $\mathbf{Q}$ corresponding to $\chi_{n-i+1}, i=1, \ldots, n$.

\section{S2 The model}

We will use the following notation:

- $\mathbf{Y} \in \mathbb{R}^{n \times m}$ is a matrix of gene expression data for $m$ genes in $n$ samples. The $i$ th column of $\mathbf{Y}$ is denoted $y_{i} \in \mathbb{R}^{n}$ and corresponds to the vector of expression values for gene $i$. We assume that the data in each sample are centred, $\sum_{i=1}^{m} y_{i}=0 \in \mathbb{R}^{n}$.

- $\mathbf{Z} \in \mathbb{R}^{n \times d}$ is a matrix of values for $d$ known confounders in the same $n$ samples. The $k$ th column of $\mathbf{Z}$ is denoted $z_{k} \in \mathbb{R}^{n}$ and corresponds to the data for confounding factor $k$.

- $\mathbf{X} \in \mathbb{R}^{n \times p}$ is a matrix of values for $p$ latent variables to be determined in the same $n$ samples. The $j$ th column of $\mathbf{X}$ is denoted $x_{j} \in \mathbb{R}^{n}$. 
To identify the hidden correlation structure of the expression data, we assume a linear relationship between expression levels and the known and latent variables, with random noise added:

$$
y_{i}=\mathbf{Z} v_{i}+\mathbf{X} w_{i}+\epsilon_{i}
$$

where $v_{i} \in \mathbb{R}^{d}$ and $w_{i} \in \mathbb{R}^{p}$ are jointly normally distributed random vectors,

$$
p\left(\left[\begin{array}{c}
v_{i} \\
w_{i}
\end{array}\right]\right)=\mathcal{N}\left(0,\left[\begin{array}{cc}
\mathbf{B} & \mathbf{D} \\
\mathbf{D}^{T} & \mathbf{A}
\end{array}\right]\right)
$$

with $\mathbf{B} \in \mathbb{R}^{d \times d}, \mathbf{D} \in \mathbb{R}^{d \times p}$ and $\mathbf{A}=\operatorname{diag}\left(\alpha_{1}^{2}, \ldots, \alpha_{p}^{2}\right)$, such that

$$
\Psi=\left[\begin{array}{cc}
\mathbf{B} & \mathbf{D} \\
\mathbf{D}^{T} & \mathbf{A}
\end{array}\right]
$$

is a positive definite matrix; the errors $\epsilon_{i} \in \mathbb{R}^{n}$ are assumed to be independent and normally distributed,

$$
p\left(\epsilon_{i}\right)=\mathcal{N}\left(0, \sigma^{2} \mathbb{1}\right)
$$

Note that our aim is to identify variance components shared across genes, and hence $\sigma^{2}$ is assumed to be the same for all $i$. By assumption, the errors are also independent of the effect sizes, and hence we can write

$$
p\left(\left[\begin{array}{c}
v_{i} \\
w_{i} \\
\epsilon_{i}
\end{array}\right]\right)=\mathcal{N}\left(0,\left[\begin{array}{ccc}
\mathbf{B} & \mathbf{D} & 0 \\
\mathbf{D}^{T} & \mathbf{A} & 0 \\
0 & 0 & \sigma^{2} \mathbb{1}
\end{array}\right]\right)
$$

By Lemma $1, y_{i}$ is normally distributed with distribution

$$
p\left(y_{i}\right)=\mathcal{N}(0, \mathbf{K})=\frac{1}{(2 \pi)^{\frac{n}{2}} \sqrt{\operatorname{det}(\mathbf{K})}} \exp \left(-\frac{1}{2}\left\langle y_{i}, \mathbf{K}^{-1} y_{i}\right\rangle\right)
$$

where

$$
\mathbf{K}=\left[\begin{array}{lll}
\mathbf{Z} & \mathbf{X} & \mathbb{1}
\end{array}\right]\left[\begin{array}{ccc}
\mathbf{B} & \mathbf{D} & 0 \\
\mathbf{D}^{T} & \mathbf{A} & 0 \\
0 & 0 & \sigma^{2} \mathbb{1}
\end{array}\right]\left[\begin{array}{c}
\mathbf{Z}^{T} \\
\mathbf{X}^{T} \\
\mathbb{1}
\end{array}\right]=\mathbf{Z B Z}^{T}+\mathbf{Z D X}^{T}+\mathbf{X D}^{T} \mathbf{Z}+\mathbf{X} \mathbf{A} \mathbf{X}^{T}+\sigma^{2} \mathbb{1},
$$

and we used the notation $\langle u, v\rangle=u^{T} v$ to denote the inner product between two vectors in $\mathbb{R}^{n}$.

Defining matrices $\mathbf{V} \in \mathbb{R}^{d \times m}$ and $\mathbf{W} \in \mathbb{R}^{p \times m}$, whose columns are the random effect vectors $v_{i}$ and $w_{i}$, respectively, eq. (S2) can be written in matrix notation as

$$
\mathbf{Y}=\mathbf{Z V}+\mathbf{X W}+\epsilon
$$

Under the assumption that the columns $y_{i}$ of $\mathbf{Y}$ are independent samples of the distribution (S5), the likelihood of observing $\mathbf{Y}$ given covariate data $\mathbf{Z}$, (unknown) latent variable data $\mathbf{X}$ and values for the hyper-parameters $\Theta=\left\{\sigma^{2}, \mathbf{A}, \mathbf{B}, \mathbf{D}\right\}$, is given by

$$
p(\mathbf{Y} \mid \mathbf{Z}, \mathbf{X}, \Theta)=\prod_{i=1}^{m} p\left(y_{i} \mid 0, \mathbf{K}\right) \text {. }
$$


Note that in standard mixed-model calculations, the distribution (S5) is often arrived at by integrating out the random effects. This is equivalent to application of Lemma 1.

To conclude, the log-likelihood is, upto an additive constant, and divided by half the number of genes:

$$
\mathcal{L}=-\frac{2}{m}\left[\frac{m}{2} \log \operatorname{det}(\mathbf{K})+\frac{1}{2} \sum_{i=1}^{m}\left\langle y_{i}, \mathbf{K}^{-1} y_{i}\right\rangle\right]=-\log \operatorname{det}(\mathbf{K})-\operatorname{tr}\left(\mathbf{K}^{-1} \mathbf{C}\right),
$$

where

$$
\mathbf{C}=\frac{\mathbf{Y} \mathbf{Y}^{T}}{m}
$$

is the empirical covariance matrix.

\section{S3 Systematic effects on the mean}

Eq. (S2) only considers random effects, which leads to a model for studying systematic effects on the covariance between samples. We could also include fixed effects to model systematic effects on mean expression level. However, by centering the data, $\sum_{i=1}^{m} y_{i}=0$, the maximumlikelihood estimate of such fixed effects is always zero. To see this, let $\mathbf{T} \in \mathbb{R}^{n \times c}$ be a matrix of $c$ covariates with fixed effects $\beta \in \mathbb{R}^{c}$ shared across genes (we are only interested in discovering systematic biases in the data). Then the minus log-likelihood (2) becomes

$$
\mathcal{L}=\log \operatorname{det}(\mathbf{K})+\frac{1}{m} \sum_{i=1}^{m}\left\langle y_{i}-\mathbf{T} \beta, \mathbf{K}^{-1}\left(y_{i}-\mathbf{T} \beta\right)\right\rangle
$$

Optimizing with respect to $\beta$ leads to the equation

$$
\hat{\beta}=\left(\mathbf{T}^{T} \mathbf{K}^{-1} \mathbf{T}\right)^{-1} \mathbf{T}^{T} \bar{y}
$$

where

$$
\bar{y}=\frac{1}{m} \sum_{i=1}^{m} y_{i}=0
$$

\section{S4 Solution of the model without latent variables}

We start by considering the problem of finding the maximum-likelihood solution in the absence of any latent variables, i.e. minimizing eq. (2) with

$$
\mathbf{K}=\mathbf{Z B Z} \mathbf{Z}^{T}+\sigma^{2} \mathbb{1}
$$

with respect to $\mathbf{B}$ and $\sigma^{2}$.

Note first of all that we may assume the set of confounding factors $\left\{z_{1}, \ldots, z_{d}\right\}$ to be linearly independent, because if not, the expression in eq. (S2) can be rearranged in terms of a linearly independent subset of factors whose coefficients are still normally distributed due to 
elementary properties of the multivariate normal distribution, see for instance the proof of Lemma 5 below. Linear independence of $\left\{z_{1}, \ldots, z_{d}\right\}$ implies that we must have $d \leq n$ and $\operatorname{rank}(\mathbf{Z})=d$.

The singular value decomposition allows to decompose $\mathbf{Z}$ as $\mathbf{Z}=\mathbf{U} \Gamma \mathbf{V}^{T}$, where $\mathbf{U} \in \mathbb{R}^{n \times n}$, $\mathbf{U}^{T} \mathbf{U}=\mathbf{U U}^{T}=\mathbb{1}, \boldsymbol{\Gamma} \in \mathbb{R}^{n \times d}$ diagonal with $\gamma_{k}^{2} \equiv \Gamma_{k k}>0$ for $k \in\{1, \ldots, d\}$ [this uses $\operatorname{rank}(\mathbf{Z})=d]$, and $\mathbf{V} \in \mathbb{R}^{d \times d}, \mathbf{V}^{T} \mathbf{V}=\mathbf{V} \mathbf{V}^{T}=\mathbb{1}$. There is also a 'thin' $\operatorname{SVD}, \mathbf{Z}=\mathbf{U}_{1} \boldsymbol{\Gamma}_{1} \mathbf{V}^{T}$, where $\mathbf{U}_{1} \in \mathbb{R}^{n \times d}, \mathbf{U}_{1}^{T} \mathbf{U}_{1}=\mathbb{1}, \boldsymbol{\Gamma}_{1} \in \mathbb{R}^{d \times d}$ diagonal with diagonal elements $\gamma_{k}^{2}$. In block matrix notation, $\mathbf{U}=\left(\mathbf{U}_{1}, \mathbf{U}_{2}\right)$ and

$$
\mathbf{Z}=\left(\begin{array}{ll}
\mathbf{U}_{1} & \mathbf{U}_{2}
\end{array}\right)\left(\begin{array}{c}
\boldsymbol{\Gamma}_{1} \\
0
\end{array}\right) \mathbf{V}
$$

Note that unitarity of $\mathbf{U}$ implies $\mathbf{U}_{1}^{T} \mathbf{U}_{2}=0$.

Denote by $\mathcal{H}_{Z}$ the space spanned by the columns (i.e. covariate vectors) of $\mathbf{Z}$. The projection matrix $\mathbf{P}_{Z}$ onto $\mathcal{H}_{Z}$ is given by

$$
\mathbf{P}_{\mathbf{Z}}=\mathbf{Z}\left(\mathbf{Z}^{T} \mathbf{Z}\right)^{-1} \mathbf{Z}^{T}=\mathbf{U}_{1} \boldsymbol{\Gamma}_{1} \mathbf{V}^{T}\left(\mathbf{V} \Gamma_{1}^{-2} \mathbf{V}^{T}\right) \mathbf{V} \boldsymbol{\Gamma}_{1} \mathbf{U}_{1}^{T}=\mathbf{U}_{1} \mathbf{U}_{1}^{T}
$$

Using the basis of column vectors of $\mathbf{U}$, we can write any matrix $\mathbf{M} \in \mathbb{R}^{n \times n}$ as a partitioned matrix

$$
\mathbf{U}^{T} \mathbf{M U}=\left(\begin{array}{ll}
\mathbf{M}_{11} & \mathbf{M}_{12} \\
\mathbf{M}_{21} & \mathbf{M}_{22}
\end{array}\right)
$$

where

$$
\mathbf{M}_{i j}=\mathbf{U}_{i}^{T} \mathbf{M} \mathbf{U}_{j}
$$

The following results for partitioned matrices are derived easily or can be found in [25]:

$$
\begin{aligned}
\operatorname{tr}(\mathbf{M}) & =\operatorname{tr}\left(\mathbf{M}_{11}\right)+\operatorname{tr}\left(\mathbf{M}_{22}\right) \\
\operatorname{det}(\mathbf{M}) & =\operatorname{det}\left(\mathbf{M}_{11}-\mathbf{M}_{12} \mathbf{M}_{22}^{-1} \mathbf{M}_{21}\right) \operatorname{det}\left(\mathbf{M}_{22}\right)
\end{aligned}
$$

Using this notation, the following result solves the model without latent variables:

Theorem 1. Let $\mathbf{C} \in \mathbb{R}^{n \times n}$ be a positive definite matrix such that

$$
\lambda_{\min }\left(\mathbf{C}_{11}\right)>\frac{\operatorname{tr}\left(\mathbf{C}_{22}\right)}{n-d}
$$

where $\lambda_{\min }(\cdot)$ denotes the smallest eigenvalue of a matrix. Then the maximum-likelihood solution

$$
\hat{\mathbf{K}}=\underset{\left\{\mathbf{K}: \mathbf{K}=\mathbf{Z} \mathbf{B Z} \mathbf{Z}^{T}+\sigma^{2} \mathbb{1}\right\}}{\operatorname{argmin}} \log \operatorname{det} \mathbf{K}+\operatorname{tr}\left(\mathbf{K}^{-1} \mathbf{C}\right),
$$

subject to $\mathbf{B}$ being positive definite and $\sigma^{2} \geq 0$, is given by

$$
\begin{aligned}
\hat{\mathbf{B}} & =\mathbf{V} \boldsymbol{\Gamma}_{1}^{-1}\left(\mathbf{C}_{11}-\hat{\sigma}^{2} \mathbb{1}\right) \boldsymbol{\Gamma}_{1}^{-1} \mathbf{V}^{T} \\
\hat{\sigma}^{2} & =\frac{\operatorname{tr}\left(\mathbf{C}_{22}\right)}{n-d}
\end{aligned}
$$


Proof. Using eq. (S7), we can write

$$
\begin{aligned}
\mathbf{K}=\mathbf{Z B Z} \mathbf{Z}^{T}+\sigma^{2} \mathbb{1} & =\mathbf{U}_{1} \boldsymbol{\Gamma}_{1} \mathbf{V}^{T} \mathbf{B} \mathbf{V} \boldsymbol{\Gamma}_{1} \mathbf{U}_{1}^{T}+\sigma^{2}\left(\mathbf{U}_{1} \mathbf{U}_{1}^{T}+\mathbf{U}_{2} \mathbf{U}_{2}^{T}\right) \\
& =\mathbf{U}_{1} \boldsymbol{\Gamma}_{1} \mathbf{V}^{T}\left(\mathbf{B}+\sigma^{2} \mathbf{V} \boldsymbol{\Gamma}_{1}^{-2} \mathbf{V}^{T}\right) \mathbf{V} \boldsymbol{\Gamma}_{1} \mathbf{U}_{1}^{T}+\sigma^{2} \mathbf{U}_{2} \mathbf{U}_{2}^{T} .
\end{aligned}
$$

Hence, in the block matrix notation (S8), we have

$$
\begin{aligned}
& \mathbf{K}_{11}=\boldsymbol{\Gamma}_{1} \mathbf{V}^{T}\left(\mathbf{B}+\sigma^{2} \mathbf{V} \boldsymbol{\Gamma}_{1}^{-2} \mathbf{V}^{T}\right) \mathbf{V} \boldsymbol{\Gamma}_{1} \\
& \mathbf{K}_{22}=\sigma^{2} \mathbb{1} \\
& \mathbf{K}_{12}=\mathbf{K}_{21}=0 .
\end{aligned}
$$

It follows that

$$
\mathbf{K}^{-1}=\left(\begin{array}{cc}
\mathbf{K}_{11}^{-1} & 0 \\
0 & \mathbf{K}_{22}^{-1}
\end{array}\right)
$$

and, using eqs. (S9) and (S10),

$$
\begin{aligned}
& \log \operatorname{det}(\mathbf{K})=\log \operatorname{det}\left(\mathbf{K}_{11}\right)+\log \operatorname{det}\left(\mathbf{K}_{22}\right)=\log \operatorname{det}\left(\mathbf{K}_{11}\right)+(n-d) \log \left(\sigma^{2}\right) \\
& \operatorname{tr}\left(\mathbf{K}^{-1} \mathbf{C}\right)=\operatorname{tr}\left(\mathbf{K}_{11}^{-1} \mathbf{C}_{11}\right)+\operatorname{tr}\left(\mathbf{K}_{22}^{-1} \mathbf{C}_{22}\right)=\operatorname{tr}\left(\mathbf{K}_{11}^{-1} \mathbf{C}_{11}\right)+\frac{\operatorname{tr}\left(\mathbf{C}_{22}\right)}{\sigma^{2}} .
\end{aligned}
$$

Let $\mathbf{C}_{11}$ have eigenvalues $\lambda_{1} \geq \cdots \geq \lambda_{d}$ with corresponding eigenvectors $u_{1}, \ldots, u_{d} \in \mathbb{R}^{d}$. Applying Lemma 2 to the term $\operatorname{tr}\left(\mathbf{K}_{11}^{-1} \mathbf{C}_{11}\right)$, it follows that for the minimizer $\hat{\mathbf{K}}, \hat{\mathbf{K}}_{11}$ must have eigenvalues $\kappa_{1} \geq \cdots \geq \kappa_{d}$ with the same eigevectors $u_{1}, \ldots, u_{d}$ as $\mathbf{C}_{11}$. Expressing the minus log-likelihood in terms of these eigenvalues results in

$$
\mathcal{L}(\hat{\mathbf{K}})=\sum_{i=1}^{d} \log \left(\kappa_{i}\right)+\sum_{i=1}^{d} \kappa_{i}^{-1} \lambda_{i}+(n-d) \log \left(\sigma^{2}\right)+\frac{\operatorname{tr}\left(\mathbf{C}_{22}\right)}{\sigma^{2}} .
$$

Minimizing with respect to the parameters $\kappa_{i}$ and $\sigma^{2}$ (i.e., setting their derivatives to zero) results in the solution $\hat{\kappa}_{i}=\lambda_{i}$ for all $i$ and $\hat{\sigma}^{2}=\frac{\operatorname{tr}\left(\mathbf{C}_{22}\right)}{n-d}$. In other words, $\hat{\mathbf{K}}_{11}$ has the same eigenvalues and eigenvectors as $\mathbf{C}_{11}$, that is,

$$
\hat{\mathbf{K}}_{11}=\mathbf{C}_{11} \text {. }
$$

This equation is satisfied if

$$
\hat{\mathbf{B}}+\hat{\sigma}^{2} \mathbf{V} \boldsymbol{\Gamma}_{1}^{-2} \mathbf{V}^{T}=\mathbf{V} \boldsymbol{\Gamma}_{1}^{-1} \mathbf{C}_{11} \boldsymbol{\Gamma}_{1}^{-1} \mathbf{V}^{T}
$$

or

$$
\hat{\mathbf{B}}=\mathbf{V} \boldsymbol{\Gamma}_{1}^{-1}\left(\mathbf{C}_{11}-\hat{\sigma}^{2} \mathbb{1}\right) \boldsymbol{\Gamma}_{1}^{-1} \mathbf{V}^{T}
$$

$\hat{\mathbf{B}}$ is positive definite if and only if for all $v \in \mathbb{R}^{d}$

$$
0<\langle v, \hat{\mathbf{B}} v\rangle=\left\langle w,\left(\mathbf{C}_{11}-\hat{\sigma}^{2} \mathbb{1}\right) w\right\rangle,
$$

where $w=\Gamma_{1} \mathbf{V} v$. Because $\mathbf{V}$ is unitary and $\boldsymbol{\Gamma}_{1}$ diagonal with strictly positive elements, $\langle v, \hat{\mathbf{B}} v\rangle>0$ for all $v \in \mathbb{R}^{d}$ if and only if $\left\langle w,\left(\mathbf{C}_{11}-\hat{\sigma}^{2} \mathbb{1}\right) w\right\rangle>0$ for all $w \in \mathbb{R}^{d}$, or

$$
0<\min _{w \in \mathbb{R}^{d}} \frac{\left\langle w, \mathbf{C}_{11} w\right\rangle}{\langle w, w\rangle}-\hat{\sigma}^{2}=\lambda_{\min }\left(\mathbf{C}_{11}\right)-\hat{\sigma}^{2}
$$


Eq. (S11) is a condition on the amount of variation in $\mathbf{Y}$ explained by the confounders $\mathbf{Z}$, with $\lambda_{\min }\left(\mathbf{C}_{11}\right)$ being (proportional to) the minimum amount of variation explained by any of the dimensions spanned by the columns of $\mathbf{Z}$, and $\frac{1}{n-d} \operatorname{tr}\left(\mathbf{C}_{22}\right)$ being the average amount of variation explained by the dimensions orthogonal to the columns of $\mathbf{Z}$. Failure of this condition simply means that there must be other, latent variables that explain more variation than the known ones, which is precisely what we are seeking to detect.

A useful special case of Theorem 1 occurs when the number of confounders equals one. In this case, we are seeking maximum-likelihood solutions for $\mathbf{K}$ of the form

$$
\mathbf{K}=\beta^{2} z z^{T}+\sigma^{2} \mathbb{1},
$$

where $z \in \mathbb{R}^{n}$ is the confounding data vector. Let $\gamma^{2}=\|z\|^{2}$ and $u=\frac{1}{\gamma} z$. Then $\mathbf{P}_{z}=u u^{T}$ is the projection matrix onto $z, \mathbf{C}_{11}=\langle u, \mathbf{C} u\rangle$, and $\operatorname{tr}\left(\mathbf{C}_{22}\right)=\operatorname{tr}\left(\left(1-\mathbf{P}_{z}\right) \mathbf{C}\right)=\operatorname{tr}(\mathbf{C})-\langle u, \mathbf{C} u\rangle$. By Theorem 1, we have

$$
\begin{aligned}
\hat{\beta}^{2} & =\frac{1}{\gamma^{2}}\left\{\langle u, \mathbf{C} u\rangle-\frac{\operatorname{tr}\left(\left[1-\mathbf{P}_{z}\right] \mathbf{C}\right)}{n-1}\right\} \\
& =\frac{1}{\gamma^{2}}\left\{\frac{n}{n-1}\langle u, \mathbf{C} u\rangle-\frac{\operatorname{tr}(\mathbf{C})}{n-1}\right\} \\
\hat{\sigma}^{2} & =\frac{\operatorname{tr}\left(\left[1-\mathbf{P}_{z}\right] \mathbf{C}\right)}{n-1}=\frac{\operatorname{tr}(\mathbf{C})-\langle u, \mathbf{C} u\rangle}{n-1},
\end{aligned}
$$

provided

$$
\langle u, \mathbf{C} u\rangle>\frac{\operatorname{tr}(\mathbf{C})}{n}
$$

\section{S5 Solution of the model without known covariates}

Next, consider a model without known covariates, i.e. with posterior sample covariance matrix $\mathbf{K}=\mathbf{K}_{X}\left(\left\{\alpha_{j}, x_{j}\right\}\right)+\sigma^{2} \mathbb{1}$, where

$$
\mathbf{K}_{X}\left(\left\{\alpha_{j}, x_{j}\right\}\right)=\sum_{j=1}^{p} \alpha_{j}^{2} x_{j} x_{j}^{T} .
$$

This model is equivalent to probabilistic principal component analysis [19,20], and its maximumlikelihood solution is given by the first $p$ eigenvectors or principal components with largest eigenvalues of $\mathbf{C}$. Here we present a more direct proof of this fact than what can be found in the literature.

Lemma 3. Without loss of generality, we may assume that the latent variables have unit norm, are linearly independent, and are mutually orthogonal.

Proof. If the latent variables do not have unit norm, define $c_{j}=\left\|x_{j}\right\|^{-1}, \alpha_{j}^{\prime}=\alpha_{j} / c_{j}$ and $x_{j}^{\prime}=c_{j} x_{j}$ for all $j$. It follows immediately that $\left\|x_{j}^{\prime}\right\|=1$ and

$$
\mathbf{K}_{X}\left(\left\{\alpha_{j}, x_{j}\right\}\right)=\mathbf{K}_{X}\left(\left\{\alpha_{j}^{\prime}, x_{j}^{\prime}\right\}\right)
$$


Next assume that the latent variables are not linearly independent, i.e. that $\operatorname{rank}\left(\mathbf{K}_{X}\right)=r<$ $p$. Because $\mathbf{K}_{X}$ is a symmetric matrix, we must have $\mathbf{K}_{X}=\sum_{l=1}^{r} t_{l} t_{l}^{T}$ for some set of linearly independent vectors $t_{l} \in \mathbb{R}^{n}$. Define $\alpha_{l}^{\prime}=\left\|t_{l}\right\|$ and $x_{l}^{\prime}=t_{l} /\left\|t_{l}\right\|$. Then $x_{l}^{\prime}$ has unit norm and

$$
\mathbf{K}_{X}\left(\left\{\alpha_{l}^{\prime}, x_{l}^{\prime}\right\}\right)=\mathbf{K}_{X}\left(\left\{\alpha_{j}, x_{j}\right\}\right) .
$$

Finally, recall that

$$
\mathbf{K}_{X}\left(\left\{\alpha_{j}, x_{j}\right\}\right)=\mathbf{X} \mathbf{A} \mathbf{X}^{T}=\left(\mathbf{X A}^{\frac{1}{2}}\right)\left(\mathbf{X A}^{\frac{1}{2}}\right)^{T},
$$

where $\mathbf{A}=\operatorname{diag}\left(\alpha_{1}^{2}, \ldots, \alpha_{p}^{2}\right)$. Because we may now assume that $\operatorname{rank}(\mathbf{X})=p$, and because $\alpha_{j}>0$ for all $j$, the matrix $\mathbf{X A}^{\frac{1}{2}}$ has singular value decomposition

$$
\mathbf{X} \mathbf{A}^{\frac{1}{2}}=\mathbf{U} \Xi \mathbf{V}^{T}
$$

with $\mathbf{U} \in \mathbb{R}^{n \times p}, \mathbf{U}^{T} \mathbf{U}=\mathbb{1}, \Xi \in \mathbb{R}^{p \times p}$ diagonal with diagonal elements $\Xi_{j j}=\xi_{j}>0$, and $\mathbf{V} \in \mathbb{R}^{p \times p}, \mathbf{V}^{T} \mathbf{V}=\mathbf{V} \mathbf{V}^{T}=\mathbb{1}$. Hence

$$
\mathbf{K}_{X}\left(\left\{\alpha_{j}, x_{j}\right\}\right)=\mathbf{U} \Xi^{2} \mathbf{U}^{T}=\sum_{j=1}^{p} \xi_{j}^{2} u_{j} u_{j}^{T}=\mathbf{K}_{X}\left(\left\{\xi_{j}, u_{j}\right\}\right),
$$

with $u_{j}$ the orthonormal columns of $\mathbf{U},\left\langle u_{j}, u_{j}^{\prime}\right\rangle=\left(\mathbf{U}^{T} \mathbf{U}\right)_{j j^{\prime}}=\delta_{j, j^{\prime}}$.

We will also need the following simple result:

Lemma 4. Let $\lambda_{1} \geq \lambda_{2} \geq \cdots \geq \lambda_{n}>0$ be a decreasing sequence of positive numbers, and let $1 \leq p<n$. If there exists $j>p$ such that $\lambda_{p}>\lambda_{j}$, then

$$
\lambda_{p}>\frac{1}{n-p} \sum_{j=p+1}^{n} \lambda_{j}
$$

Proof. Eq. (S16) follows from

$$
\lambda_{p}-\frac{1}{n-p} \sum_{j=p+1}^{n} \lambda_{j}=\frac{1}{n-p} \sum_{j=p+1}^{n}\left(\lambda_{p}-\lambda_{j}\right)>0,
$$

because each term on the r.h.s. is non-negative, and at least one is strictly positive.

Theorem 2. Let $\mathbf{C} \in \mathbb{R}^{n \times n}$ be a positive definite matrix with eigenvalues $\lambda_{1} \geq \cdots \geq \lambda_{n}$ and corresponding eigenvectors $u_{1}, \ldots, u_{n}$, and let either $p=n$ or $1 \leq p<n$ such that there exists $j>p$ with $\lambda_{p}>\lambda_{j}$. Then the maximum-likelihood solution

$$
\hat{\mathbf{K}}=\underset{\left\{\mathbf{K}: \mathbf{K}=\mathbf{X} \mathbf{A} \boldsymbol{X}^{T}+\sigma^{2} \mathbb{1}\right\}}{\operatorname{argmin}} \log \operatorname{det} \mathbf{K}+\operatorname{tr}\left(\mathbf{K}^{-1} \mathbf{C}\right),
$$

is given by

$$
\begin{aligned}
\hat{x}_{j} & =u_{j} \\
\hat{\alpha}_{j}^{2} & =\lambda_{j}-\hat{\sigma}^{2} \\
\hat{\sigma}^{2} & =\frac{1}{n-p} \sum_{j=p+1}^{n} \lambda_{j} .
\end{aligned}
$$


Proof. By Lemma 3, we can assume that $\mathbf{X}$ has orthonormal columns, and hence there exist $\mathbf{V} \in \mathbb{R}^{n \times(n-p)}$ such that $\mathbf{Q}=(\mathbf{X}, \mathbf{V}) \in \mathbb{R}^{n \times n}$ is unitary, $\mathbf{Q}^{T} \mathbf{Q}=\mathbf{Q Q}^{T}=\mathbb{1}$. Hence $\mathbf{K}=$ $\mathbf{X} \mathbf{A} \mathbf{X}^{T}+\sigma^{2} \mathbb{1}$ has the spectral decomposition

$$
\mathbf{K}=\left(\begin{array}{ll}
\mathbf{X} & \mathbf{V}
\end{array}\right)\left(\begin{array}{cc}
\mathbf{A}^{2}+\sigma^{2} \mathbb{1} & 0 \\
0 & \sigma^{2} \mathbb{1}
\end{array}\right)\left(\begin{array}{l}
\mathbf{X}^{T} \\
\mathbf{V}^{T}
\end{array}\right)
$$

and hence

$$
\mathbf{K}^{-1}=\sum_{j=1}^{p} \frac{1}{\alpha_{j}^{2}+\sigma^{2}} x_{j} x_{j}^{T}+\frac{1}{\sigma^{2}} \sum_{l=1}^{n-p} v_{l} v_{l}^{T},
$$

where $v_{l} \in \mathbb{R}^{n}$ are the columns of $\mathbf{V}$.

Assume that the $\alpha_{j}^{2}$ are ordered, $\alpha_{1}^{2} \geq \cdots \geq \alpha_{p}^{2}$. Applying von Neumann's Lemma 2 gives

$$
\begin{aligned}
\mathcal{L} & =\log \operatorname{det}(\mathbf{K})+\operatorname{tr}\left(\mathbf{K}^{-1} \mathbf{C}\right) \\
& \geq \sum_{j=1}^{p} \log \left(\alpha_{j}^{2}+\sigma^{2}\right)+(n-p) \log \left(\sigma^{2}\right)+\sum_{j=1}^{p} \frac{\lambda_{j}}{\alpha_{j}^{2}+\sigma^{2}}+\sum_{j=p+1}^{n} \frac{\lambda_{j}}{\sigma^{2}},
\end{aligned}
$$

with equality if and only if

$$
\begin{aligned}
& x_{j}=u_{j} \text { for } j=1, \ldots, p \\
& v_{l}=u_{p+l} \text { for } l=1, \ldots, n-p
\end{aligned}
$$

Hence, independent of the values for $\alpha_{j}$, the maximum-likelihood latent variables are the eigenvectors of $\mathbf{C}$ corresponding to the $p$ largest eigenvalues. Minimizing eq, (S17) w.r.t. $\alpha_{j}^{2}$ and $\sigma^{2}$ then gives

$$
\begin{aligned}
\alpha_{j}^{2} & =\lambda_{j}-\sigma^{2} \\
\sigma^{2} & =\frac{1}{n-p} \sum_{j=p+1}^{N} \lambda_{j} .
\end{aligned}
$$

By Lemma $4, \alpha_{j}^{2}>0$ for all $j$.

Note that plugging the maximum-likelihood values in the likelihood function gives

$$
\mathcal{L}_{\min }=\sum_{j=1}^{p} \log \left(\lambda_{j}\right)+(n-p) \log \left(\frac{1}{n-p} \sum_{j=p+1}^{n} \lambda_{j}\right)+n
$$

Either $p$ can be set a priori small enough such that condition (S16) is satisfied, or else the value of $p$ with smallest $\mathcal{L}_{\text {min }}$ satisfying this condition can be found easily from eq. (S18).

Note also that in the models of $[19,20]$, uniform prior variances are assumed $\left(\alpha_{1}^{2}=\cdots=\right.$ $\alpha_{p}^{2}=1$ ), such that $\mathbf{X}$ is defined upto an arbitrary rotation, because $\mathbf{X} \mathbf{X}^{T}=(\mathbf{X} \mathbf{R})(\mathbf{X R})^{T}$ for any rotation matrix $\mathbf{R}$. In our model, there is no such rotational freedom (if $\mathbf{A}$ is assumed to be diagonal), except if $\mathbf{C}$ has eigenvalues with multiplicities greater than one, when there is some freedom to choose the corresponding eigenvectors. 


\section{S6 Solution of the full model}

\section{S6.1 Orthogonality of known and hidden confounders}

Lemma 5. Without loss of generality, we may assume that the latent variables are orthogonal to the known confounders:

$$
\mathbf{X}^{T} \mathbf{Z}=\mathbf{Z} \mathbf{X}^{T}=0 .
$$

Proof. As in Section S4, let $\mathbf{P}_{Z}$ again be the projection matrix on the space spanned by the known covariates $z_{k}$ (i.e. the columns of $\mathbf{Z}$ ). For any choice of latent variables $x_{j}$, we have

$$
x_{j}=\mathbf{P}_{Z} x_{j}+\left(1-\mathbf{P}_{Z}\right) x_{j}=\sum_{k=1}^{d} m_{k j} z_{k}+\tilde{x}_{j}
$$

for some matrix of linear coefficients $\mathbf{M}=\left(m_{k q}\right) \in \mathbb{R}^{d \times p}$, and with $\left\langle s_{k}, \tilde{x}_{j}\right\rangle=0$ for all $k$. Or, in matrix notation

$$
\mathbf{X}=\mathbf{Z M}+\tilde{\mathbf{X}}, \quad \text { with } \quad \tilde{\mathbf{X}}^{T} \mathbf{Z}=\mathbf{Z}^{T} \tilde{\mathbf{X}}=0
$$

Plugging this in eq. (S2), results in

$$
y_{i}=\mathbf{Z} \tilde{v}_{i}+\tilde{\mathbf{X}} w_{i}+\epsilon_{i}
$$

where $\tilde{v}_{i}=v_{i}+\mathbf{M} w_{i}$. Hence

$$
\left[\begin{array}{c}
\tilde{v}_{i} \\
w_{i} \\
\epsilon_{i}
\end{array}\right]=\left[\begin{array}{lll}
\mathbb{1} & M & 0 \\
0 & \mathbb{1} & 0 \\
0 & 0 & \mathbb{1}
\end{array}\right]\left[\begin{array}{c}
v_{i} \\
w_{i} \\
\epsilon_{i}
\end{array}\right]
$$

and hence, using Lemma 1, it follows that

$$
p\left(\left[\begin{array}{c}
\tilde{v}_{i} \\
w_{i} \\
\epsilon_{i}
\end{array}\right]\right)=\mathcal{N}\left(0,\left[\begin{array}{ccc}
\mathbf{B}+\mathbf{M D}^{T}+\mathbf{D} \mathbf{M}^{T}+\mathbf{M} \mathbf{A} \mathbf{M}^{T} & \mathbf{D}+\mathbf{A} \mathbf{M}^{T} & 0 \\
\mathbf{D}^{T}+\mathbf{M A} & \mathbf{A} & 0 \\
0 & 0 & \sigma^{2} \mathbb{1}
\end{array}\right]\right) .
$$

This is still of exactly the same form as eq. (S4). Hence model (S20) is identical to model (S2), but has hidden covariates orthogonal to the known covariates.

Note that we can parameterize the model with hidden variables orthogonal to the known confounders, $\mathbf{Z}^{T} \mathbf{X}=0$, but only if we allow the covariances of their effects on gene expression, $\operatorname{Cov}\left(v_{i}, w_{i}\right)=\mathbf{D}$, to be non-zero. Equivalently, we can parameterize the model such that the random effects of hidden variables are statistically independent of the effects of the known confounders, $\operatorname{Cov}\left(v_{i}, w_{i}\right)=0$, but only if we allow the hidden variables to overlap with the known confounders, $\mathbf{Z}^{T} \mathbf{X} \neq 0$. Mathematically, the choice of orthogonal hidden factors will be much more convenient.

Note also that a transformation to orthogonal hidden factors always induces non-zero covariances among the known confounders via the term $\mathbf{M A \mathbf { M } ^ { T }}$. Hence an important difficulty with the model where $\mathbf{B}$ is assumed to be diagonal, as used in [15], comes from the fact that non-orthogonal hidden variables are needed to model off-diagonal covariances between the known confounders. It is much more intuitive to model these directly by assuming a general covariance matrix. 


\section{S6.2 Restricted maximum-likelihood solution for the latent variables}

Lemma 6. Without loss of generality, we may assume that the latent variables have unit norm, are linearly independent, and are mutually orthogonal.

Proof. The proof is identical to the proof of Lemma 3 - it is straightforward to verify that the transformation to orthonormal variables also do not change the form of the off-diagonal term $\mathbf{Z D X}^{T}$ in the covariance matrix $\mathbf{K}$, but merely lead to a reparameterization of the matrix D.

To solve the full model, we follow an approach similar to the standard restricted maximumlilelihood method for linear mixed models [21,23]: we write the negative log-likelihood function $\mathcal{L}=\log \operatorname{det}(\mathbf{K})+\operatorname{tr}\left(\mathbf{K}^{-1} \mathbf{C}\right)$ as a sum

$$
\mathcal{L}=\mathcal{L}_{1}+\mathcal{L}_{2}
$$

where $\mathcal{L}_{2}$ will be the log-likelihood restricted to the subspace orthogonal to the known confounders $\mathbf{Z}$. We will estimate the latent variables $\mathbf{X}$ and their effect covariances $\mathbf{A}$ by maximizing $\mathcal{L}_{2}$, and estimate the effect covariances $\mathbf{B}$ and $\mathbf{D}$ involving the known confounders by maximizing $\mathcal{L}_{1}$. Solving for the latent variables on a restricted subspace is motivated by the observation that if $y \in \mathbb{R}^{n}$ is a sample from the model (S2), that is, $p(y)=\mathcal{N}(0, \mathbf{K})$, then

$$
\mathbf{U}_{2} \mathbf{U}_{2}^{T} y=\mathbf{U}_{2} \mathbf{U}_{2}^{T} \mathbf{Z} v+\mathbf{U}_{2} \mathbf{U}_{2}^{T} \mathbf{X} w+\mathbf{U}_{2} \mathbf{U}_{2}^{T} \epsilon=\mathbf{X} w+\epsilon^{\prime} .
$$

In other words, restricted to the subspace orthogonal to $\mathbf{Z}$, the general model becomes a probablistic PCA model where all variation in the data is explained by the latent variables.

To obtain the decomposition (S21), we partition $y \in \mathbb{R}^{n}$ as $y=\left(y_{1}, y_{2}\right)^{T}$, where $y_{1}=\mathbf{U}_{1}^{T} y \in$ $\mathbb{R}^{d}$ and $y_{2}=\mathbf{U}_{2}^{T} y \in \mathbb{R}^{n-d}$, and write

$$
p(y)=p\left(y_{1}, y_{2}\right)=p\left(y_{1} \mid y_{2}\right) p\left(y_{2}\right),
$$

or

$$
\log p(y)=\log p\left(y_{1}, y_{2}\right)=\log p\left(y_{1} \mid y_{2}\right)+\log p\left(y_{2}\right)
$$

Hence

$$
\mathcal{L}=-\frac{2}{m} \sum_{i=1}^{m} \log p\left(y_{i}\right)=\underbrace{-\frac{2}{m} \sum_{i=1}^{m} \log p\left(y_{i 1} \mid y_{i 2}\right)}_{\mathcal{L}_{1}} \underbrace{-\frac{2}{m} \sum_{i=1}^{m} \log p\left(y_{i 2}\right)}_{\mathcal{L}_{2}}
$$

Using standard results for the marginal and conditional distributions of a multivariate Gaussian, we have

$$
\begin{aligned}
p\left(y_{2}\right) & =\mathcal{N}\left(0, \mathbf{K}_{22}\right) \\
p\left(y_{1} \mid y_{2}\right) & =\mathcal{N}\left(\mathbf{K}_{12} \mathbf{K}_{22}^{-1} y_{2},\left(\mathbf{K}_{11}-\mathbf{K}_{12} \mathbf{K}_{22}^{-1} \mathbf{K}_{21}\right)\right),
\end{aligned}
$$


where we used the partitioned matrix notation of eq. (S8). In particular,

$$
\begin{aligned}
\mathcal{L}_{2} & =\log \operatorname{det}\left(\mathbf{K}_{22}\right)+\frac{1}{m} \sum_{i=1}^{m}\left\langle\mathbf{U}_{2}^{T} y_{i}, \mathbf{K}_{22}^{-1} \mathbf{U}_{2}^{T} y_{i}\right\rangle \\
& =\log \operatorname{det}\left(\mathbf{K}_{22}\right)+\frac{1}{m} \sum_{i=1}^{m} \operatorname{tr}\left(\mathbf{K}_{22}^{-1} \mathbf{U}_{2}^{T} y_{i} y_{i}^{T} \mathbf{U}_{2}\right) \\
& =\log \operatorname{det}\left(\mathbf{K}_{22}\right)+\operatorname{tr}\left(\mathbf{K}_{22}^{-1} \mathbf{C}_{22}\right) .
\end{aligned}
$$

Note that $\mathbf{K}_{22}=\mathbf{U}_{2}^{T} \mathbf{X} \mathbf{A} \mathbf{X}^{T} \mathbf{U}_{2}^{T}+\sigma^{2} \mathbb{1}$, and hence $\mathcal{L}_{2}$ depends only on $\mathbf{X}, \mathbf{A}$ and $\sigma^{2}$. The restricted maximum likelihood solution for the latent variables follows immediately:

Theorem 3. Let $\hat{\mathbf{X}} \in \mathbb{R}^{n \times p}, \hat{A} \in \mathbb{R}^{d \times d}$, and $\hat{\sigma}^{2}$ be the solution of

$$
\left\{\hat{\mathbf{X}}, \hat{\mathbf{A}}, \hat{\sigma}^{2}\right\}=\underset{\mathbf{X}, \mathbf{A}, \sigma^{2}}{\operatorname{argmin}} \mathcal{L}_{2}\left(\mathbf{X}, \mathbf{A}, \sigma^{2}\right),
$$

where the minimum is taken over all $\mathbf{X}$ with $\mathbf{X}^{T} \mathbf{Z}=0$, and all positive definite diagonal matrices $\hat{\mathbf{A}}$. If there exists $j>p$ such that $\lambda_{p}>\lambda_{j}$, then

$$
\begin{aligned}
\hat{\mathbf{X}} & =\mathbf{U}_{2} \mathbf{W}_{p} \\
\hat{\mathbf{A}} & =\operatorname{diag}\left(\lambda_{1}-\hat{\sigma}^{2}, \ldots, \lambda_{p}-\hat{\sigma}^{2}\right) \\
\hat{\sigma}^{2} & =\frac{1}{n-d-p} \sum_{j=p+1}^{n-d} \lambda_{j}
\end{aligned}
$$

where $\lambda_{1} \geq \lambda_{2} \geq \cdots \geq \lambda_{n-d}$ are the sorted eigenvalues of $\mathbf{C}_{22}$ with corresponding eigenvectors $w_{1}, \ldots, w_{n-d} \in \mathbb{R}^{n-d}$, and $\mathbf{W}_{p}=\left(w_{1}, \ldots, w_{p}\right) \in \mathbb{R}^{(n-d) \times p}$ is the matrix with the first $p$ eigenvectors of $\mathbf{C}_{22}$ as columns.

Proof. Defining $\tilde{\mathbf{X}}=\mathbf{U}_{2}^{T} \mathbf{X} \in \mathbb{R}^{(n-d) \times p}$, we have $\mathbf{K}_{22}=\tilde{\mathbf{X}} \mathbf{A} \tilde{\mathbf{X}}^{T}+\sigma^{2} \mathbb{1}$, and $\mathcal{L}_{2}$ becomes precisely the minus log-likelihood of the model without known covariates (Section S5), as a function of the latent variables $\tilde{\mathbf{X}}$ on the reduced $(n-d)$-dimensional space orthogonal to the known confounders Z . Hence by Theorem 2,

$$
\begin{aligned}
& \hat{\tilde{\mathbf{X}}}=\mathbf{W}_{p} \\
& \hat{\mathbf{A}}=\operatorname{diag}\left(\lambda_{1}-\sigma^{2}, \ldots, \lambda_{p}-\sigma^{2}\right),
\end{aligned}
$$

where $\lambda_{1} \geq \lambda_{2} \geq \cdots \geq \lambda_{n-d}$ are the sorted eigenvalues of $\mathbf{C}_{22}$ and $\mathbf{W}_{p} \in \mathbb{R}^{(n-d) \times p}$ is the matrix having the corresponding first $p$ eigenvectors as columns. Note that $\hat{\mathbf{A}}$ is positive definite by Lemma 4 and the assumption that there exists $j>p$ such that $\lambda_{p}>\lambda_{j}$. It remains to 'pull-back' $\tilde{\mathbf{X}}$ to the original $n$-dimensional space, using the orthogonality condition (S19):

$$
\hat{\mathbf{X}}=\left(\mathbf{U}_{1} \mathbf{U}_{1}^{T}+\mathbf{U}_{2} \mathbf{U}_{2}^{T}\right) \hat{\mathbf{X}}=\mathbf{U}_{2} \mathbf{U}_{2}^{T} \hat{\mathbf{X}}=\mathbf{U}_{2} \hat{\tilde{\mathbf{X}}}=\mathbf{U}_{2} \mathbf{W}_{p} .
$$

This proves eqs. (S22) and (S23). 


\section{S6.3 Solution for the variance parameters given the latent variables}

With $\hat{\mathbf{X}}, \hat{\mathbf{A}}$ and $\hat{\sigma}^{2}$ determined by the minimization of $\mathcal{L}_{2}$ in Theorem $3, \mathcal{L}_{2}\left(\hat{\mathbf{X}}, \hat{\mathbf{A}}, \hat{\sigma}^{2}\right)$ is constant in terms of the parameters $\mathbf{B}$ and $\mathbf{D}$ that remain to be optimized. Hence optimizing $\mathcal{L}_{1}$ with respect to these parameters is the same as optimizing the total negative log-likelihood $\mathcal{L}\left(\hat{\mathbf{X}}, \hat{\mathbf{A}}, \mathbf{B}, \mathbf{D}, \hat{\sigma}^{2}\right)$ w.r.t. B and D. We have:

Theorem 4. Let $\hat{\mathbf{B}} \in \mathbb{R}^{d \times d}$ and $\hat{D} \in \mathbb{R}^{d \times(n-d)}$ be the solution of

$$
\{\hat{\mathbf{B}}, \hat{\mathbf{D}}\}=\underset{\mathbf{B}, \mathbf{D}}{\operatorname{argmin}} \mathcal{L}_{1}\left(\hat{\mathbf{X}}, \hat{\mathbf{A}}, \mathbf{B}, \mathbf{D}, \hat{\sigma}^{2}\right)=\underset{\mathbf{B}, \mathbf{D}}{\operatorname{argmin}} \mathcal{L}\left(\hat{\mathbf{X}}, \hat{\mathbf{A}}, \mathbf{B}, \mathbf{D}, \hat{\sigma}^{2}\right),
$$

subject to the constraint that $\mathbf{B}$ and $\mathbf{B}-\mathbf{D} \hat{\mathbf{A}}^{-1} \mathbf{D}^{T}$ are positive definite. If

$$
\lambda_{\min }\left(C_{11}\right)>\hat{\sigma}^{2}
$$

then

$$
\begin{aligned}
\hat{\mathbf{B}} & =\mathbf{V} \boldsymbol{\Gamma}_{1}^{-1}\left(\mathbf{C}_{11}-\hat{\sigma}^{2} \mathbb{1}\right) \boldsymbol{\Gamma}_{1}^{-1} \mathbf{V}^{T} \\
\hat{\mathbf{D}} & =\mathbf{V} \boldsymbol{\Gamma}_{1}^{-1} \mathbf{C}_{12} \mathbf{W}_{p}
\end{aligned}
$$

where as before

$$
\mathbf{Z}=\left(\begin{array}{ll}
\mathbf{U}_{1} & \mathbf{U}_{2}
\end{array}\right)\left(\begin{array}{c}
\boldsymbol{\Gamma}_{1} \\
0
\end{array}\right) \mathbf{V}^{T}
$$

is the singular value decomposition of $\mathbf{Z}$, and $\mathbf{W}_{p}=\left(w_{1}, \ldots, w_{p}\right) \in \mathbb{R}^{(n-d) \times p}$ is the matrix with the first $p$ eigenvectors of $\mathbf{C}_{22}$ as columns.

Proof. Note that the conditions $\mathbf{B}$ and $\mathbf{B}-\mathbf{D} \hat{\mathbf{A}}^{-1} \mathbf{D}^{T}$ positive definite are to ensure that the $\operatorname{matrix}\left(\begin{array}{cc}\mathbf{B} & \mathbf{D} \\ \mathbf{D}^{T} & \hat{\mathbf{A}}\end{array}\right)$ is positive definite. Next note that with $\hat{\mathbf{X}}^{T}$ known, the covariance matrix $\mathbf{K}$ can be written as

$$
\mathbf{K}=\left(\begin{array}{ll}
\mathbf{Z} & \hat{\mathbf{X}}
\end{array}\right)\left(\begin{array}{cc}
\mathbf{B} & \mathbf{D} \\
\mathbf{D}^{T} & \hat{\mathbf{A}}
\end{array}\right)\left(\begin{array}{l}
\mathbf{Z}^{T} \\
\hat{\mathbf{X}}^{T}
\end{array}\right)+\hat{\sigma}^{2} \mathbb{1}
$$

Hence the total log-likelihood is identical to the model with known covariates $\tilde{\mathbf{Z}}=\left(\begin{array}{ll}\mathbf{Z} \quad \hat{\mathbf{X}}\end{array}\right)$ and no latent variables (Section S4). The unconstrained maximizing solution (that is, where A and $\sigma^{2}$ are also optimized) for the model with known covariates $\tilde{\mathbf{Z}}$ is given by Theorem 1 . Due to $\hat{\mathbf{X}}^{T} \mathbf{Z}=0$ and the definition of $\hat{\mathbf{X}}$, the singular value decomposition of $\tilde{\mathbf{Z}}$ is given by

$$
\tilde{\mathbf{Z}}=\left(\begin{array}{lll}
\mathbf{U}_{1} & \hat{\mathbf{X}} & \mathbf{U}_{3}
\end{array}\right)\left(\begin{array}{cc}
\Gamma_{1} & 0 \\
0 & \mathbb{1} \\
0 & 0
\end{array}\right)\left(\begin{array}{cc}
\mathbf{V}^{T} & 0 \\
0 & \mathbb{1}
\end{array}\right),
$$

where the columns of $\mathbf{U}_{3} \in \mathbb{R}^{n \times(n-d-p)}$ span the space orthogonal to the columns of $\tilde{\mathbf{Z}}$. Hence the unconstrained solution, can be written as (cf. eqs. (S13)-(S14))

$$
\begin{aligned}
\left(\begin{array}{cc}
\hat{\mathbf{B}} & \hat{\mathbf{D}} \\
\hat{\mathbf{D}}^{T} & \hat{\mathbf{A}}^{\prime}
\end{array}\right) & =\left(\begin{array}{ll}
\mathbf{V} & 0 \\
0 & \mathbb{1}
\end{array}\right)\left(\begin{array}{cc}
\boldsymbol{\Gamma}_{1}^{-1} & 0 \\
0 & \mathbb{1}
\end{array}\right)\left(\begin{array}{l}
\mathbf{U}_{1}^{T} \\
\hat{\mathbf{X}}^{T}
\end{array}\right)\left(\mathbf{C}-\hat{\sigma}^{\prime 2} \mathbb{1}\right)\left(\begin{array}{cc}
\mathbf{U}_{1} & \hat{\mathbf{X}}
\end{array}\right)\left(\begin{array}{cc}
\boldsymbol{\Gamma}_{1}^{-1} & 0 \\
0 & \mathbb{1}
\end{array}\right)\left(\begin{array}{cc}
\mathbf{V}^{T} & 0 \\
0 & \mathbb{1}
\end{array}\right) \\
\hat{\sigma}^{\prime 2} & =\frac{\operatorname{tr}\left(\mathbf{U}_{3}^{T} \mathbf{C} \mathbf{U}_{3}\right)}{n-d-p}
\end{aligned}
$$


First note that $\hat{\sigma}^{\prime 2}=\hat{\sigma}^{2}$, because we can write $\mathbf{U}_{3}=\mathbf{U}_{2} \mathbf{W}_{\sim p}$, where $\mathbf{W}_{\sim p} \in \mathbb{R}^{(n-d) \times(n-d-p)}$ is the matrix with the $n-d-p$ last eigenvectors of $\mathbf{C}_{22}$.

Working out the block matrix product results in:

$$
\begin{aligned}
\hat{\mathbf{B}} & =\mathbf{V} \boldsymbol{\Gamma}_{1}^{-1} \mathbf{U}_{1}^{T}\left(\mathbf{C}-\hat{\sigma}^{2} \mathbb{1}\right) \mathbf{U}_{1} \boldsymbol{\Gamma}_{1}^{-1} \mathbf{V}^{T}=\mathbf{V} \boldsymbol{\Gamma}_{1}^{-1}\left(\mathbf{C}_{11}-\hat{\sigma}^{2} \mathbb{1}\right) \boldsymbol{\Gamma}_{1}^{-1} \mathbf{V}^{T} \\
\hat{\mathbf{D}} & =\mathbf{V} \boldsymbol{\Gamma}_{1}^{-1} \mathbf{U}_{1}^{T} \mathbf{C} \hat{\mathbf{X}}=\mathbf{V} \boldsymbol{\Gamma}_{1}^{-1} \mathbf{U}_{1}^{T} \mathbf{C} \mathbf{U}_{2} \mathbf{W}_{p}=\mathbf{V} \boldsymbol{\Gamma}_{1}^{-1} \mathbf{C}_{12} \mathbf{W}_{p} \\
\hat{\mathbf{A}}^{\prime} & =\hat{\mathbf{X}}^{T}\left(\mathbf{C}-\hat{\sigma}^{2} \mathbb{1}\right) \hat{\mathbf{X}}=\mathbf{W}_{p}^{T} \mathbf{U}_{2}^{T}\left(\mathbf{C}-\hat{\sigma}^{2} \mathbb{1}\right) \mathbf{U}_{2} \mathbf{W}_{p}=\mathbf{W}_{p}^{T}\left(\mathbf{C}_{22}-\hat{\sigma}^{2} \mathbb{1}\right) \mathbf{W}_{p} \\
& =\operatorname{diag}\left(\lambda_{1}-\hat{\sigma}^{2}, \ldots, \lambda_{p}-\hat{\sigma}^{2}\right)
\end{aligned}
$$

Hence, also the estimate $\hat{\mathbf{A}}^{\prime}=\hat{\mathbf{A}}$. Because the unconstrained optimization of $\mathcal{L}$ given $\hat{\mathbf{X}}$ results in the same estimate for $\mathbf{A}$ and $\sigma^{2}$ as the intial constrained optimization where these parameters were given, it follows that also the estimates of $\mathbf{B}$ and $\mathbf{D}$ must be the same:

$$
\{\hat{\mathbf{B}}, \hat{\mathbf{D}}\}=\underset{\mathbf{B}, \mathbf{D}}{\operatorname{argmin}} \mathcal{L}\left(\mathbf{B}, \mathbf{D} \mid \hat{\mathbf{X}}, \hat{\mathbf{A}}, \hat{\sigma}^{2}\right)=\underset{\mathbf{B}, \mathbf{D}}{\operatorname{argmin}} \min _{\mathbf{A}, \sigma^{2}} \mathcal{L}\left(\mathbf{A}, \mathbf{B}, \mathbf{D}, \sigma^{2} \mid \hat{\mathbf{X}}\right) .
$$

\section{S6.4 LVREML maximizes the variance explained}

It is tempting to ask whether the combined solution from Theorems 3 and 4 optimizes the total likelihood among all possible $p$-dimensional sets of latent variables. To address this problem, let $\mathbf{X} \in \mathbb{R}^{n \times p}$ be an arbitrary matrix of latent variables whose columns are normalized, mutually orthogonal and orthogonal to the columns of $\mathbf{Z}, \mathbf{X}^{T} \mathbf{X}=\mathbb{1}$ and $\mathbf{X}^{T} \mathbf{Z}=0$. Because $\mathbf{U}_{2}$ is only defined upto a rotation, we can always choose

$$
\mathbf{U}_{2}=\left(\begin{array}{ll}
\mathbf{X} & \mathbf{Q}
\end{array}\right)
$$

with $\mathbf{Q} \in \mathbb{R}^{n \times(n-d-p)}$ satisfying $\mathbf{Q}^{T} \mathbf{Q}=\mathbb{1}, \mathbf{Q}^{T} \mathbf{X}=0$ and $\mathbf{Q}^{T} \mathbf{Z}=0$. From the proof of Theorem 4 we immediately obtain:

Proposition 1. Let $\mathbf{A}(\mathbf{X}) \in \mathbb{R}^{p \times p}, \mathbf{B}(\mathbf{X}) \in \mathbb{R}^{d \times d}, \mathbf{D}(\mathbf{X}) \in \mathbb{R}^{d \times(n-d)}$ and $\sigma^{2}(\mathbf{X})>0$ be the solution of

$$
\left\{\mathbf{A}(\mathbf{X}), \mathbf{B}(\mathbf{X}), \mathbf{D}(\mathbf{X}), \sigma^{2}(\mathbf{X})\right\}=\underset{\mathbf{A}, \mathbf{B}, \mathbf{D}, \sigma^{2}}{\operatorname{argmin}} \mathcal{L}\left(\mathbf{A}, \mathbf{B}, \mathbf{D}, \sigma^{2} \mid \mathbf{X}\right) .
$$

Then

$$
\begin{aligned}
\mathbf{B}(\mathbf{X}) & =\mathbf{V} \boldsymbol{\Gamma}_{1}^{-1}\left(\mathbf{C}_{11}-\hat{\sigma}^{2} \mathbb{1}\right) \boldsymbol{\Gamma}_{1}^{-1} \mathbf{V}^{T} \\
\mathbf{D}(\mathbf{X}) & =\mathbf{V} \boldsymbol{\Gamma}_{1}^{-1} \mathbf{U}_{1}^{T} \mathbf{C} \mathbf{X} \\
\mathbf{A}(\mathbf{X}) & =\mathbf{X}^{T}\left(\mathbf{C}-\hat{\sigma}^{2} \mathbb{1}\right) \mathbf{X} \\
\sigma^{2}(\mathbf{X}) & =\frac{\operatorname{tr}\left(\mathbf{Q}^{T} \mathbf{C} \mathbf{Q}\right)}{n-d-p}
\end{aligned}
$$


Plugging these values into the negative log-likelihood function results in a function that depends only on $\mathbf{X}$ :

Proposition 2. Let $\mathbf{X} \in \mathbb{R}^{n \times p}$ be an arbitrary choice of latent variables with associated maximumlikelihood estimates for the covariance parameters given by Proposition 1. Then, upto an additive constant

$$
\mathcal{L}_{\mathbf{X}}=\log \operatorname{det}\left(\mathbf{X}^{T}\left[\mathbf{C}-\mathbf{C U}_{1}\left(\mathbf{U}_{1}^{T} \mathbf{C} \mathbf{U}_{1}\right) \mathbf{U}_{1}^{T} \mathbf{C}\right] \mathbf{X}\right)+(n-d-p) \log \left(\hat{\sigma}^{2}(\mathbf{X})\right)
$$

Proof. Recall from Theorem 2 that the maximum-likelihood estimate for $\mathbf{K}$ given $\mathbf{X}$ and its associated maximum-likelihood parameters estimates is given by

$$
\hat{\mathbf{K}}(\mathbf{X})=\left(\begin{array}{ccc}
\mathbf{U}_{1}^{T} \mathbf{C U}_{1} & \mathbf{U}_{1}^{T} \mathbf{C X} & 0 \\
\mathbf{X}^{T} \mathbf{C} \mathbf{U}_{1} & \mathbf{X}^{T} \mathbf{C X} & 0 \\
0 & 0 & \hat{\sigma}^{2} \mathbb{1}
\end{array}\right)
$$

while the covariance matrix $\mathbf{C}$ can be written as

$$
\mathbf{C}=\left(\begin{array}{ccc}
\mathbf{U}_{1}^{T} \mathbf{C U}_{1} & \mathbf{U}_{1}^{T} \mathbf{C X} & \mathbf{U}_{1}^{T} \mathbf{C Q} \\
\mathbf{X}^{T} \mathbf{C} \mathbf{U}_{1} & \mathbf{X}^{T} \mathbf{C X} & \mathbf{X}^{T} \mathbf{C Q} \\
\mathbf{Q C} \mathbf{U}_{1} & \mathbf{Q C X} & \mathbf{Q}^{T} \mathbf{C Q}
\end{array}\right)
$$

Hence

$$
\begin{aligned}
\mathcal{L}_{\mathbf{X}} & =\mathcal{L}(\hat{\mathbf{K}}(\mathbf{X}))=\log \operatorname{det}(\hat{\mathbf{K}}(\mathbf{X}))+\operatorname{tr}\left(\hat{\mathbf{K}}(\mathbf{X})^{-1} \mathbf{C}\right) \\
& =\log \operatorname{det}\left(\begin{array}{ll}
\mathbf{U}_{1}^{T} \mathbf{C} \mathbf{U}_{1} & \mathbf{U}_{1}^{T} \mathbf{C X} \\
\mathbf{X}^{T} \mathbf{C} \mathbf{U}_{1} & \mathbf{X}^{T} \mathbf{C X}
\end{array}\right)+(n-d-p) \log \left(\hat{\sigma}^{2}\right)+(d+p)+\frac{\operatorname{tr}\left(\mathbf{Q}^{T} \mathbf{C} \mathbf{Q}\right)}{\hat{\sigma}^{2}} \\
& =\log \operatorname{det}\left(\begin{array}{ll}
\mathbf{U}_{1}^{T} \mathbf{C} \mathbf{U}_{1} & \mathbf{U}_{1}^{T} \mathbf{C X} \\
\mathbf{X}^{T} \mathbf{C} \mathbf{U}_{1} & \mathbf{X}^{T} \mathbf{C X}
\end{array}\right)+(n-d-p) \log \left(\hat{\sigma}^{2}\right)+(d+p)+(n-d-p)
\end{aligned}
$$

Using equation (S10) for the determinant of a partitioned matrix, we have

$$
\begin{aligned}
\log \operatorname{det}\left(\begin{array}{ll}
\mathbf{U}_{1}^{T} \mathbf{C} \mathbf{U}_{1} & \mathbf{U}_{1}^{T} \mathbf{C X} \\
\mathbf{X}^{T} \mathbf{C} \mathbf{U}_{1} & \mathbf{X}^{T} \mathbf{C X}
\end{array}\right) & =\log \operatorname{det}\left(\mathbf{U}_{1}^{T} \mathbf{C} \mathbf{U}_{1}\right)+\log \operatorname{det}\left(\mathbf{X}^{T} \mathbf{C X}-\mathbf{X}^{T} \mathbf{C} \mathbf{U}_{1}\left(\mathbf{U}_{1}^{T} \mathbf{C} \mathbf{U}_{1}\right)^{-1} \mathbf{U}_{1} \mathbf{C X}\right) \\
& =\log \operatorname{det}\left(\mathbf{U}_{1}^{T} \mathbf{C} \mathbf{U}_{1}\right)+\log \operatorname{det}\left(\mathbf{X}^{T}\left[\mathbf{C}-\mathbf{C U}_{1}\left(\mathbf{U}_{1}^{T} \mathbf{C U}_{1}\right)^{-1} \mathbf{U}_{1}^{T} \mathbf{C}\right] \mathbf{X}\right) .
\end{aligned}
$$

Ignoring the constants $\log \operatorname{det}\left(\mathbf{U}_{1}^{T} \mathbf{C} \mathbf{U}_{1}\right)$ and $n$ which do not depend on $\mathbf{X}$, we obtain eq. (S28).

Due to the determinant term in eq. (S28), it is not clear whether the restricted maximumlikelihood solution $\hat{\mathbf{X}}$ of Theorem 3 (with its associated maximum-likelihood covariance parameters of Theorem 4) is the absolute minimizer of $\mathcal{L}_{\mathbf{X}}$,

$$
\hat{\mathbf{X}}=\underset{\mathbf{X} \in \mathbb{R}^{n \times p}, \mathbf{X}^{T} \mathbf{X}=\mathbb{1}, \mathbf{X}^{T} \mathbf{Z}=0}{\operatorname{argmin}} \mathcal{L} \quad ?
$$

However, we do have the following result: 
Theorem 5. The restricted maximum-likelihood solution $\hat{\mathbf{X}}$ of Theorem 3 is the set of $p$ latent variables that minimizes the residual variance among all choices of $p$ latent variables,

$$
\hat{\mathbf{X}}=\underset{\mathbf{X} \in \mathbb{R}^{n \times p}, \mathbf{X}^{T} \mathbf{X}=\mathbb{1}, \mathbf{X}^{T} \mathbf{Z}=0}{\operatorname{argmin}} \sigma^{2}(\mathbf{X})
$$

Proof. By Proposition 1 and the arguments leading up to it, we can write

$$
\operatorname{tr}\left(\mathbf{C}_{22}\right)=\operatorname{tr}\left(\mathbf{X}^{T} \mathbf{C X}\right)+\operatorname{tr}\left(\mathbf{Q}^{T} \mathbf{C} \mathbf{Q}^{T}\right)=\operatorname{tr}\left(\left(\mathbf{U}_{2}^{T} \mathbf{X}\right)^{T} \mathbf{C}_{22}\left(\mathbf{U}_{2}^{T} \mathbf{X}\right)\right)+\operatorname{tr}\left(\left(\mathbf{U}_{2}^{T} \mathbf{Q}\right)^{T} \mathbf{C}_{22}\left(\mathbf{U}_{2}^{T} \mathbf{Q}\right)\right),
$$

where as before $\mathbf{C}_{22}=\mathbf{U}_{2}^{T} \mathbf{C} \mathbf{U}_{2}$ is the restriction of $\mathbf{C}$ to the $(n-d)$-dimensional subspace orthogonal to the $d$ known covariates, and the columns of $\mathbf{U}_{2}^{T} \mathbf{X}$ and $\mathbf{U}_{2}^{T} \mathbf{Q}$ span mutually orthogonal subspaces within this $(n-d)$-dimensional space. Hence $(n-d-p) \sigma^{2}(\mathbf{X})=$ $\operatorname{tr}\left(\mathbf{Q}^{T} \mathbf{C} \mathbf{Q}^{T}\right)$ is the trace of $\mathbf{C}_{22}$ over the residual $(n-d-p)$-dimensional space orthogonal to the latent variables, within the subspace orthogonal to the $d$ known covariates. By the Courant-Fisher min-max theorem for eigenvalues [25], the $(n-d-p)$-dimensional subspace of $\mathbb{R}^{n-d}$ with smallest trace is the subspace spanned by the eigenvectors of $\mathbf{C}_{22}$ corresponding to its $(n-d-p)$ smallest eigenvalues. By Theorem 3, this is exactly the subspace obtained by choosing $\mathbf{X}$ equal to the restricted maximum-likelihood solution $\hat{\mathbf{X}}$.

\section{S7 Selecting covariates and the latent dimension}

Two practical problems remain: how to choose the latent variable dimension parameter $p$ and which known covariates to include?

To choose $p$, we will use the following result:

\section{Lemma 7.}

$$
\operatorname{tr}(\mathbf{C})=\operatorname{tr}(\hat{\mathbf{K}})=\operatorname{tr}\left(\mathbf{Z} \hat{\mathbf{B}} \mathbf{Z}^{T}\right)+\operatorname{tr}\left(\hat{\mathbf{X}} \hat{\mathbf{A}} \hat{\mathbf{X}}^{T}\right)+n \hat{\sigma}^{2}
$$

Proof. Use Theorem 4 to compute

$$
\begin{aligned}
\operatorname{tr}(\mathbf{Z} \hat{\mathbf{B}} \mathbf{Z}) & =\operatorname{tr}\left(\mathbf{U}_{1} \boldsymbol{\Gamma}_{1} \mathbf{V}^{T}\left[\mathbf{V} \boldsymbol{\Gamma}_{1}^{-1}\left(\mathbf{C}_{11}-\hat{\sigma}^{2} \mathbb{1}\right) \boldsymbol{\Gamma}_{1}^{-1} \mathbf{V}^{T}\right] \mathbf{V} \boldsymbol{\Gamma}_{1} \mathbf{U}_{1}^{T}\right) \\
& =\operatorname{tr}\left(\mathbf{U}_{1} \mathbf{C}_{11} \mathbf{U}_{1}^{T}\right)-\hat{\sigma}^{2} \operatorname{tr}\left(\mathbf{U}_{1} \mathbf{U}_{1}^{T}\right) \\
& =\operatorname{tr}\left(\mathbf{C}_{11}\right)-d \hat{\sigma}^{2},
\end{aligned}
$$

where the last step uses the cyclical property of the trace and the fact that $\mathbf{U}_{1}^{T} \mathbf{U}_{1}=\mathbb{1}_{d}$. Likewise, we have

$$
\begin{aligned}
\operatorname{tr}(\hat{\mathbf{X}} \hat{\mathbf{A}} \hat{\mathbf{X}}) & =\operatorname{tr}\left(\mathbf{U}_{2} \mathbf{W}_{p} \operatorname{diag}\left(\lambda_{1}, \ldots, \lambda_{p}\right) \mathbf{W}_{p}^{T} \mathbf{U}_{2}^{T}\right)-\hat{\sigma}^{2} \operatorname{tr}\left(\mathbf{U}_{2} \mathbf{W}_{p} \mathbf{W}_{p}^{T} \mathbf{U}_{2}^{T}\right) \\
& =\sum_{j=1}^{p} \lambda_{j}-p \hat{\sigma}^{2} \\
& =\sum_{j=1}^{n-d} \lambda_{j}-(n-d) \hat{\sigma}^{2} \\
& =\operatorname{tr}\left(\mathbf{C}_{22}\right)-(n-d) \hat{\sigma}^{2}
\end{aligned}
$$


Hence

$$
\operatorname{tr}(\hat{\mathbf{K}})=\operatorname{tr}(\mathbf{Z} \hat{\mathbf{B} Z})+\operatorname{tr}(\hat{\mathbf{X}} \hat{\mathbf{A}} \hat{\mathbf{X}})+n \hat{\sigma}^{2}=\operatorname{tr}\left(\mathbf{C}_{11}\right)+\operatorname{tr}\left(\mathbf{C}_{22}\right)=\operatorname{tr}(\mathbf{C})
$$

Because $\mathbf{C}=\left(\mathbf{Y} \mathbf{Y}^{T}\right) / m$, the eigenvalues of $\mathbf{C}$ are (proportional to) the squared singular values of the expression data $\mathbf{Y}$. Hence $\operatorname{tr}(\mathbf{Z} \hat{\mathbf{B Z}}) / \operatorname{tr}(\mathbf{C})$ is the proportion of variation in $\mathbf{Y}$ explained by the known covariates, $\operatorname{tr}(\hat{\mathbf{X}} \hat{\mathbf{A}} \hat{\mathbf{X}}) / \operatorname{tr}(\mathbf{C})$ the proportion of variation explained by the latent variables, and $n \hat{\sigma}^{2} / \operatorname{tr}(\mathbf{C})$ is the residual variance.

Our method for determining the number of latent variables lets the user decide a priori the minimum amount of variation $\rho$ in the data that should be explained by the known and latent confounders. It follows that given $\rho$, a "target" value for $\sigma^{2}$ is

$$
\sigma^{2}(\rho)=\min \left\{\frac{(1-\rho) \operatorname{tr}(\mathbf{C})}{n}, \lambda_{\min }\left(\mathbf{C}_{11}\right)\right\}
$$

where the minimum with $\lambda_{\min }\left(\mathbf{C}_{11}\right)$ is taken to ensure that of condition (S25) remains valid. Because the eigenvalues $\lambda_{1}, \ldots, \lambda_{n-d}$ are sorted, the function

$$
f(p)=\frac{1}{n-d-p} \sum_{j=p+1}^{n-d} \lambda_{j}
$$

increases with decreasing $p$. Hence given $\rho$, we define $\hat{p}$ as

$$
\hat{p}=\min \left\{p: 0 \leq p<n-d, \lambda_{p}>\lambda_{n-d}, f(p)<\sigma^{2}(\rho)\right\},
$$

that is, we choose $\hat{p}$ to be the smallest number of latent variables that explain at least a proporition of variation $\rho$ of $\mathbf{Y}$, while guaranteeing that the conditions for all mathematical results derived in this document are valid.

Note that unless all eigenvalues of $\mathbf{C}_{22}$ are identical, $\hat{p}$ always exists. Once the desired number of latent variables $\hat{p}$ is defined, the latent factors $\hat{\mathbf{X}}$, the variance parameters $\hat{\mathbf{A}}$, and the residual variance estimate $\hat{\sigma}^{2}$ (which will be the largest possible value less than or equal to the target value $\sigma^{2}(\rho)$ ) are determined by Theorem 3. Once those are determined, the remaining covariance parameters $\hat{\mathbf{B}}$ and $\hat{\mathbf{D}}$ are determined by Theorem 4 .

A second practical problem occurs when the rank of $\mathbf{Z}$ exceeds the number of samples, such that any subset of $n$ linearly independent covariates explains all of the variation in Y. To select a more relevant subset of covariates, we rapidly screen all candidate covariates using the model with a single known covariate (Section S4) to compute the variance $\hat{\beta}^{2}$ explained by that covariate alone (eq. (S15)). We then keep only those covariates for which $\hat{\beta}^{2} \geq \theta \operatorname{tr}(\mathbf{C})$, where $\theta>0$ is the second free parameter of the method, namely the minimum amount of variation explained by a known covariate on its own. The selected covariates are ranked according to their value of $\hat{\beta}^{2}$, and a linearly independent subset is generated, starting from the covariates with highest $\hat{\beta}^{2}$. 